\title{
Theory of pattern forming systems under traveling-wave forcing
}

\author{
Sten Rüdiger ${ }^{\mathrm{a}, \mathrm{b}, *}$, Ernesto M. Nicola ${ }^{\mathrm{c}}$, Jaume Casademunt ${ }^{\mathrm{d}}$, Lorenz Kramer ${ }^{\mathrm{e}}$ \\ ${ }^{a}$ Institut für Physik, Humboldt-Universität zu Berlin, Newtonstr. 15, 12489 Berlin, Germany \\ ${ }^{\mathrm{b}}$ Hahn-Meitner Institut, Glienicker Str. 100, 14109 Berlin, Germany \\ ${ }^{c}$ Max-Planck-Institut für Physik komplexer Systeme, Nöthnitzer Straße 38, 01187 Dresden, Germany \\ ${ }^{\mathrm{d}}$ Facultat de Física, Universitat de Barcelona, Av. Diagonal 647, 08028 Barcelona, Spain \\ ${ }^{\mathrm{e}}$ Universität Bayreuth, Universitätstraße 30, D-95448 Bayreuth, Germany
}

editor: I. Procaccia

\begin{abstract}
The response of pattern forming systems to external forcing either spatial or temporal has received much attention for several decades. Combined spatio-temporal forcing has only been introduced recently, in particular in the form of a spatially resonant traveling-wave forcing [S. Rüdiger, D.G. Míguez, A.P. Muñuzuri, F. Sagués, J. Casademunt, Phys. Rev. Lett. 90 (2003) 128301]. Since then, both a series of experiments, in the context of Turing patterns in reaction-diffusion systems, and the development of the corresponding generic theory, have unveiled a wealth of new and unexpected phenomena. In this article we review these phenomena, we provide a unified and comprehensive description of them, and extend the theoretical analysis to new situations. We formulate the generic amplitude equations for different orders of spatial resonance for $1 \mathrm{~d}$ and $2 \mathrm{~d}$ patterns (stripes and hexagons). We identify and describe in detail the autonomous dynamical system which underlies the phenomenon of traveling-stripe resonance. For 1d we focus on localized solutions (kinks and pulses), their dynamics and their interaction for both 1:1 and 2:1 resonance. Specifically, we discuss the effect of wave-number mismatch (inexact resonance) combined with the non-gradient dynamics induced by the motion of the forcing, resulting in non-trivial interactions and complex spatio-temporal dynamics. Analytical results in the phase approximation are obtained, while numerical techniques are used to study the complete problem. We show that defect interaction is oscillatory with distance, allowing for the existence of locked chaotic wave trains. In $2 \mathrm{~d}$ we discuss the modulational instabilities of striped patterns and focus mostly on the emergence of hexagons induced by traveling stripe forcing, for exact 1:1 resonance. In this case, we examine in detail the complex bifurcation scenario beyond primary instabilities. We finally discuss the problem of traveling-wave forcing in the context of a specific system, the photosensitive CDIMA reaction, where most experiments have been carried out. We compare experiments and theoretical predictions and propose other experimental systems where the study could be extended. Finally, we review related work by other authors and discuss possible further developments and open questions which hold the promise of new interesting findings.
\end{abstract}

(C) 2007 Elsevier B.V. All rights reserved.

PACS: 82.40.Ck; 47.20.Ky; 47.54.+r

Keywords: Amplitude equations; Pattern formation; Kinks; Pulses; Hexagonal patterns; Turing patterns; Spatio-temporal forcing; Resonance; Modulational instabilities; Phase diffusion; Traveling-wave forcing

\footnotetext{
* Corresponding author.

E-mail address: sten.ruediger@physik.hu-berlin.de (S. Rüdiger).
} 


\section{Contents}

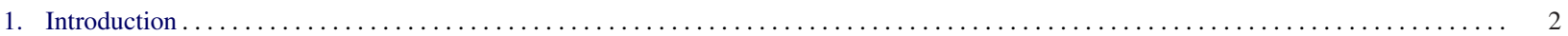

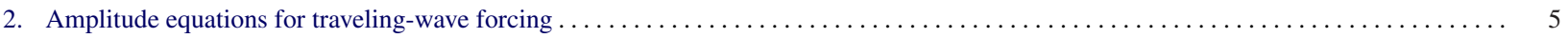

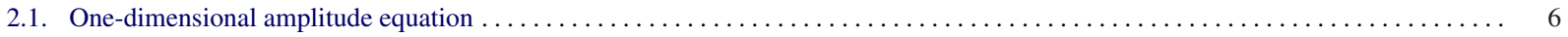

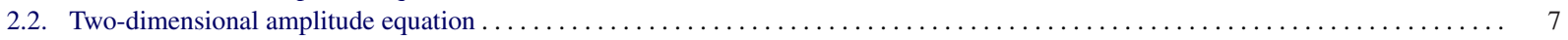

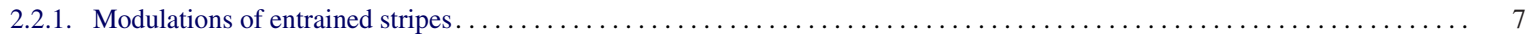

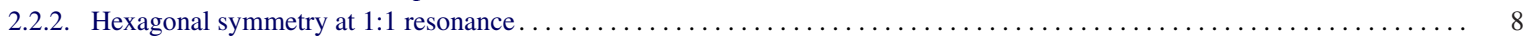

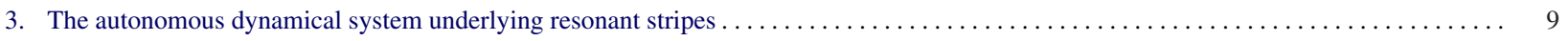

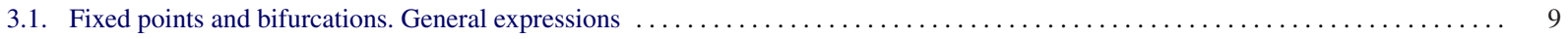

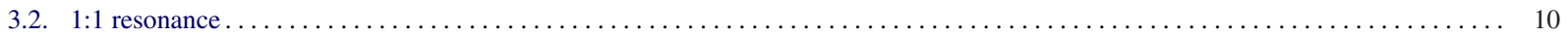

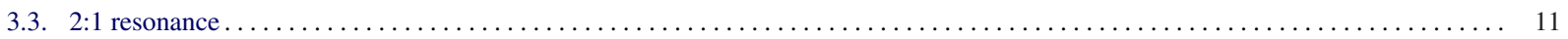

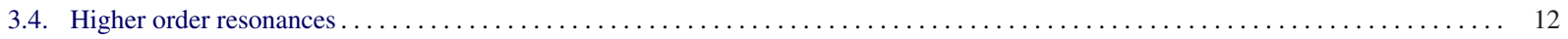

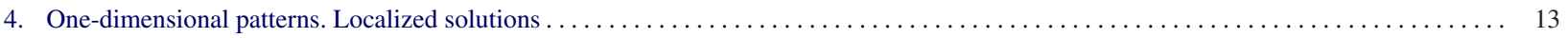

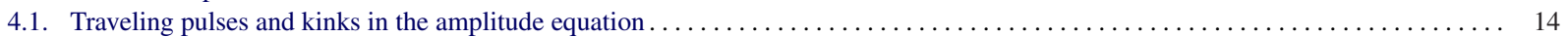

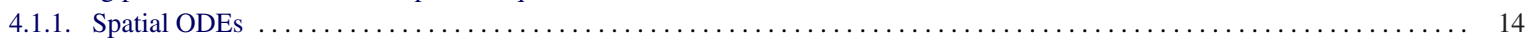

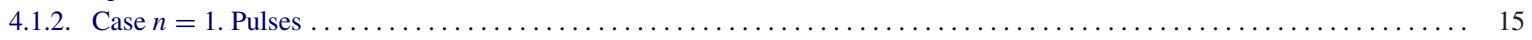

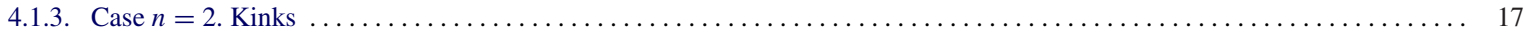

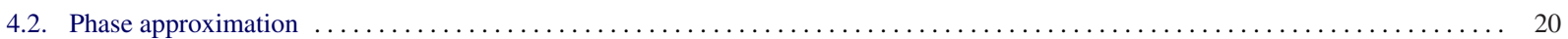

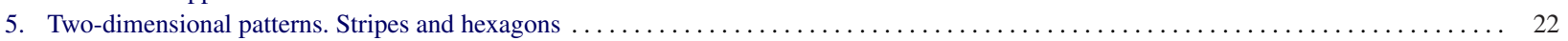

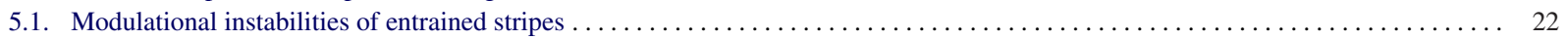

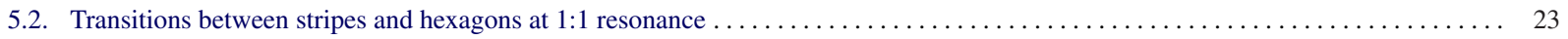

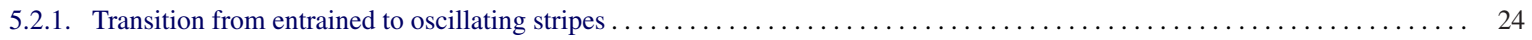

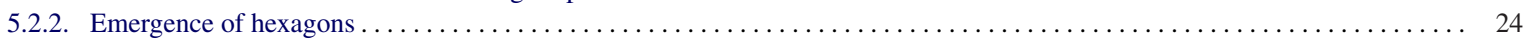

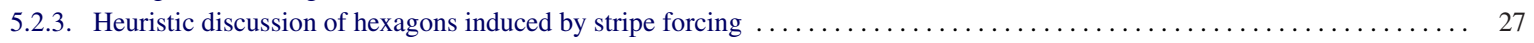

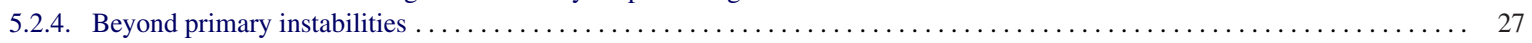

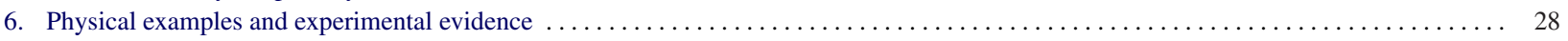

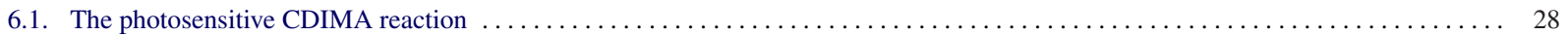

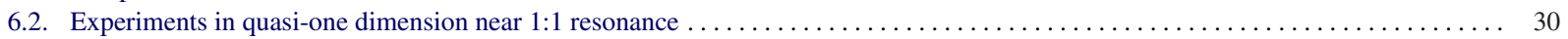

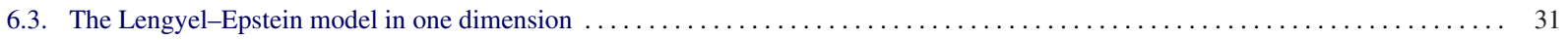

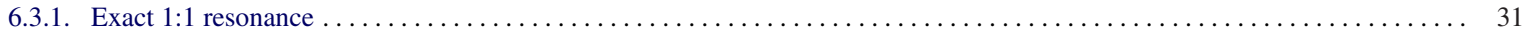

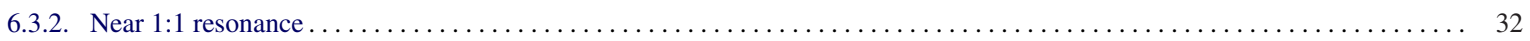

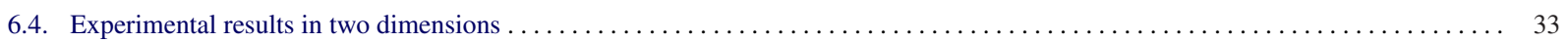

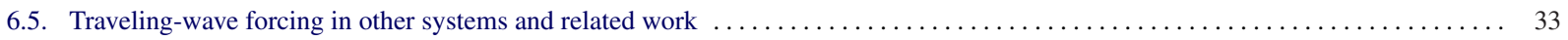

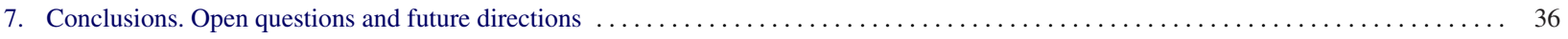

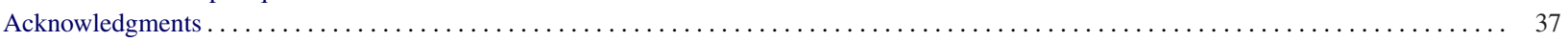

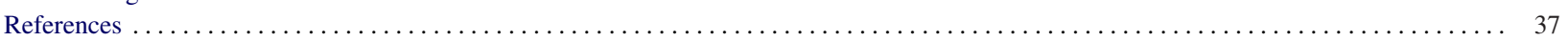

\section{Introduction}

The emergence of spatio-temporal structure in spatially extended systems out of equilibrium has attracted the attention of physicists for several decades [1,2]. We have now a fairly good understanding of the symmetry-breaking instability mechanisms leading to spontaneous pattern formation when nonlinear systems are externally driven in steady nonequilibrium conditions. Powerful tools have been developed to elucidate the universal aspects of such phenomena and provide a quantitative understanding of specific problems in physics, chemistry, material science or biology. One aspect which is less studied and which has particular relevance is the response of this class of systems to structured external forcing. The forcing not only provides a useful tool to deeply probe their inherently nonlinear behavior, but also to elucidate possible mechanisms for pattern control.

The study of pattern formation in extended systems under external forcing has indeed revealed an enormous wealth of nonlinear phenomena. Typically, one addresses the case in which a resonance phenomenon occurs between the external forcing and an intrinsic scale (temporal or spatial) if the system is driven out of equilibrium. For the case of purely temporal forcing, the study of synchronization phenomena in nonlinear oscillators and chaotic systems has uncovered interesting universal phenomena already at the level of non-extended dynamical systems but particularly in extended systems $[3,4]$. A common area of such studies is, for instance, that of nonlinear chemical reactions, either oscillatory or excitable. Here the forcing can be introduced through the sensitivity of the reactions to the illumination conditions, 
for instance in the photosensitive Belousov-Zhabotinsky reaction. In particular, the onset of spatio-temporal complexity under temporal forcing has been studied in the context of the formation of localized defects (spirals, Ising-Bloch walls, etc.). Some of the contributions in the study of pattern formation induced by temporal forcing are Refs. [5-17]. We are not addressing cases in which the forcing is itself the origin of the pattern-forming instability, such as for instance in Faraday waves $[1,18]$. In some cases, the oscillatory forcing of spatially stationary patterns has been considered [19-21].

On the other hand, the case of purely spatial forcing of pattern-forming systems has also been studied for about two decades. This line of work focused on the generation of complex spatial patterns [22-38]. Purely spatial forcing has also been applied very recently to oscillatory systems in Ref. [39].

Historically, the study of spatial forcing originated in the problem of wave-number selection in stripe forming systems. The earliest experiments on purely spatial forcing were reported in publications of Lowe and co-workers [22-24] on convecting nematic liquid crystals. Under the action of a uniform electric field, a transition to convection occurs and the director field of the liquid crystals becomes spatially periodic with a period roughly twice the layer thickness. Additionally a spatially periodic electric field is applied. By changing the layer thickness, Lowe and co-workers studied the competition between both length scales. They found periodic or commensurate states as well as incommensurate states, which consisted of arrays of defects in the pattern. These defects are regions of compression of convection rolls and can be regarded as domain walls. This work also identified a rather well-defined transition line between commensurate and the incommensurate states in the parameter space of wave-number ratio and forcing amplitude. The authors related this finding to the existence of a potential for the dynamics similar to the Frenkel-Kontorova model for equilibrium systems. These results were subsequently explained by Coullet and co-workers in Ref. [25], which in turn was followed up in a series of studies on localized solutions [26-28,30] leading for instance to the concept of spatial chaos. These contributions are among the most relevant to the one-dimensional patterns studied in the present review.

The combination of spatial and temporal forcing was proposed only recently in Ref. [40], for stationary patterns leading, already in its simplest form, to a variety of new and interesting spatio-temporal phenomena. While some of them could be expected as a natural extension of what was known for spatial and temporal forcing, many novel aspects are inherently associated to the combined spatio-temporal forcing and were completely unforeseen. A precursor of our studies of spatio-temporal forcing was reported in Ref. [41] for the more complicated case of oscillatory systems. In the present paper we will review the first steps undertaken in the exploration of spatio-temporal forcing originating in the seminal paper Ref. [40] and followed up by Refs. [42-47], all of them with a direct interplay with experiments on Turing patterns in reaction-diffusion systems. Following the above references, we will focus on the simplest case, namely that in which the external forcing takes the form of a periodic modulation of an external control parameter, which is traveling with a finite velocity. In the case of two-dimensional patterns, the forcing modulation is then assumed to consist of traveling stripes. We will also restrict to systems that, in the absence of external forcing, undergo a stationary (supercritical) bifurcation, that is, with a finite critical wave-number $k_{\mathrm{c}}$ but no finite frequency. We will refer to the case in which the control parameter $\mu$ driving the primary instability is spatially uniform and constant in time as in the unforced case. The traveling-stripe forcing is thus defined as a modulation of the control parameter $\mu$ which is spatially periodic with a wave-number $k_{f}$, assumed to be close to $n: m$ spatial resonance with the critical wavelength of the pattern forming system (i.e. $m k_{f} \simeq n k_{\mathrm{c}}$ ), see Fig. 1 . This modulation travels with a velocity $v_{f}$, hence the forcing introduces a frequency $\omega=v_{f} k_{f}$ in the system. In addition to $\omega$, the forcing is characterized by the amplitude of the modulation or strength of the forcing $\epsilon$. We will see that these two parameters compete in a non-trivial way with each other giving rise to a complex scenario of dynamical responses. In fact, if $\omega$ is small compared to the intrinsic time scales of the unforced system, the response is expected to be adiabatic. The system will respond as for the case of purely spatial forcing, although with an additional drift. In the opposite limit, for large forcing velocities, the effect of forcing modulation will be suppressed, as the system will see the time average of the forcing, i.e. the unforced case. In the intermediate range, however, one must anticipate a series of non-trivial dynamical regimes which interpolate between the behavior for purely spatial forcing with a drift, and those of the unforced problem. We will see that the competition of the forcing strength and the forcing velocity is at the root of surprising phenomena such as the emergence of hexagonal symmetry out of traveling-stripe forcing of a stripe-forming system near the 1:1 resonance. Finally, we will see that another important parameter associated to the forcing is the wave-number mismatch $q$, if the resonance is not exact. We will show that this parameter controls a symmetry breaking and is responsible for non-trivial interaction between localized solutions or defects, which will move with non-trivial velocities and generate complex spatio-temporal dynamics. The induced motion of defects and, in general, of non-trivial locked solutions is one of the 


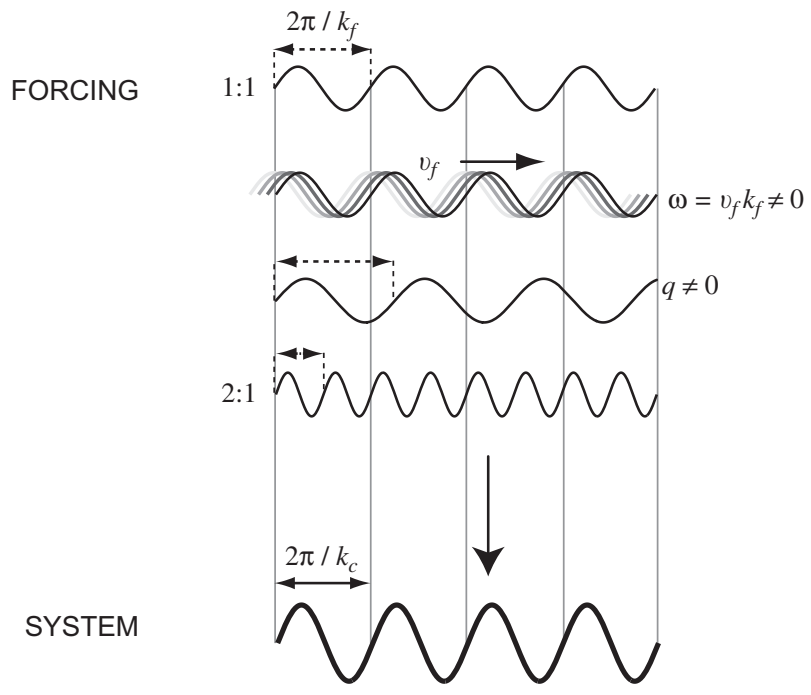

Fig. 1. Sketch of a Turing unstable system and four characteristic cases of spatially periodic forcings considered in this review.

aspects which are presumably most relevant to applications in pattern control, for instance concerning the problem of information storage and transmission, as raised in the context of Refs. [48,49].

The idea of spatio-temporal forcing has also been followed up by other authors. Traveling-stripe forcing was applied to anisotropic systems exhibiting oblique rolls and was studied in the context of liquid crystals in Ref. [50]. Its effect on the motion of spirals in excitable media was considered in Ref. [51]. A natural extension of traveling-stripe forcing to oscillatory systems supporting traveling waves has been proposed in Refs. [52-54]. Finally, other forms of spatiotemporal forcing related to the motion of parameter steps or motion of boundaries have also been proposed in Turing-like systems for instance in Refs. [55-57]. We will address these related contributions in Section 6.5.

A typical physical system for which the present discussion is relevant, and for which most of the experiments of traveling-wave forcing have been carried out so far is that of the so-called photosensitive chlorine dioxide-iodinemalonic acid (CDIMA) reaction [58,59]. This chemical reaction undergoes a Turing instability [60-63] in a certain range of parameters and can be modeled in terms of a relatively simple two-variable reaction-diffusion system. The illumination of the quasi-two-dimensional reactor acts as a control parameter which can be easily modulated at will by simple optical projection of the desired spatio-temporal pattern of light on the plane of the reaction. The idea of projecting light into a chemical reaction to probe the spatio-temporal response has been explored in the past in the case of noisy forcing [64,65], that is, with an incoherent mixture of a broad band of frequencies and wavelengths. Here we take the opposite point of view, that is, we apply a forcing with a single spatial periodicity and only the frequency inherited from the motion of the traveling-wave modulation. In the most common case of experiments performed in the parameter regime for which the unforced systems exhibits stationary stripes, the argument on the interpolation between spatial forcing and the unforced case as one increases the forcing velocity yields a rather counterintuitive prediction. In fact, in the absence of spatial mismatch, the system must evolve from a traveling-stripe state into a stationary stripe state, as the velocity is increased. The system will thus undergo a series of symmetry-breaking instabilities in order to enable this transition. In $2 \mathrm{~d}$, for instance, this may be mediated by the generation of hexagonal patterns with complex time dependence.

The present review paper will focus mostly on the theoretical framework which underlies the generic spatio-temporal phenomena behind the spatial resonance in systems under traveling-wave forcing. We will thus revisit mostly the theoretical parts of Refs. [40,42-45,47] but we will also add new analytical and numerical results and new insights into the physical significance of the results. We will thus focus our discussion on amplitude equations. In this framework, the generic character of the phenomena is naturally established and, at the same time, one can classify the possible dynamical behaviors in terms of the parameters of the amplitude equations. The connection between these parameters and the physical parameters of a particular system is then left to the specific study of each case. The CDIMA reaction defines a natural domain where many of the theoretical predictions can be tested directly, and has played indeed a central 
role in the experimental studies of the present problem. The connection between the experimental parameters and those of the reduced reaction-diffusion model is rather well known in that case and, on the other hand, the connection between the latter and those appearing in the amplitude equation has also been established [37]. Other parameter regimes of the amplitude equation however, may be relevant for different physical systems where traveling-wave forcing can be probed, for instance in nonlinear optics, Rayleigh-Bénard convection, or other reaction-diffusion systems. We will briefly address this point in Section 6.5.

The study of the full phase diagram of the corresponding amplitude equation for traveling-wave forcing is a task that goes much beyond the scope of the present review paper, even if only in one dimension. Here, we will review the general framework and give a unified description of some of the simplest scenarios of traveling-stripe forcing which have been studied in detail and which were adapted to the CDIMA system. We will complete those with new results and new views on them. Different interesting and promising directions which remain unexplored will be briefly discussed at the end.

Before we proceed to a systematic presentation, we outline the chronological development of the main steps that will be reviewed and completed in the following sections. The idea of a traveling-wave forcing in a Turing-like system in 1d was first proposed in [40] in the case of 1:1 resonance. The first theoretical predictions were then confirmed experimentally in the CDIMA reaction by the group of Santiago de Compostela [40]. In the case of exact spatial resonance the occurrence of homogeneous patterns of oscillating stripes was predicted and for slightly inexact resonance a first contact with the theory of purely spatial forcing of Ref. [25] was found, which generalized the (static) localized structures to traveling solutions. Since the experimental realization of the system was obviously two-dimensional in practice, with a traveling-stripe forcing, inherently two-dimensional phenomena were immediately revealed experimentally. Among a variety of complex spatio-temporal regimes, it came as a most remarkable surprise that a possible generic response of the system to traveling-stripe forcing was a symmetry-breaking into hexagonal patterns. Such hexagonal symmetry appeared in parameter regimes where the unforced system did not show such symmetry. The basic understanding of this phenomenon was achieved later in the context of the appropriate amplitude equation, in terms of a modification of the natural three-wave resonance mechanism that controls the generation of hexagons in the unforced system, due to the effect of the forcing of the entrained mode [42]. The corresponding amplitude equation unveiled also a complex bifurcation scenario where different dynamical modes of hexagonal and striped patterns have been identified $[43,45]$.

The case of higher-order resonances was addressed later. The complete amplitude equation for $n: m$ resonance and in the presence of a small wave-number mismatch was first reported in Ref. [44]. There the aim was at the study of kink solutions in the case of 2:1 resonance, with focus on the phase approximation. Here we will also review further work, which is in part presented in Ref. [47], where the motion of the forcing is shown to induce non-gradient dynamics, while the wave-number mismatch breaks explicitly the chiral symmetry of the domain walls. The combination of both effects yields an imperfect non-equilibrium Ising-Bloch bifurcation, where all kinks drift. Kink velocities and interactions are also studied within the generic amplitude equation framework and, for non-zero mismatch, a transition of kink interaction from monotonic to oscillatory with distance is found, leading for instance to the existence of traveling bound kink-antikink pairs and, in general, to chaotic wave trains, which generalize the spatial chaos described in [28].

The layout of this review paper is as follows. Section 2 describes the amplitude equation framework. Section 3 is concerned with general considerations of the autonomous dynamical system which is behind the phenomenon of traveling-stripe resonance. Section 4 refers to one-dimensional patterns, with focus on localized solutions and domain walls or kinks. Section 5 is concerned with two-dimensional patterns, mostly focusing on the emergence of hexagonal symmetry. Section 6 discusses explicit physical systems where the generic phenomena can be observed, including the comparison with experiments. Finally in Section 7 we conclude with our views on the most promising unexplored directions in the problem.

\section{Amplitude equations for traveling-wave forcing}

The natural mathematical framework for the study of generic pattern formation is that of amplitude equations. The general idea is that sufficiently close to the instability threshold, a natural separation of temporal and spatial scales allows for a weakly nonlinear expansion of a complex amplitude describing a smooth and slow envelope of the underlying pattern. The dynamics of this amplitude is a center manifold reduction of the original problem which captures its essential features and defines a certain degree of universality. In fact, the form of the corresponding amplitude equation 
reflects the type of bifurcation and the relevant symmetries involved, while the values of the parameters are specific to each particular problem. The connection between the physical parameters of the system undergoing a pattern forming instability and those appearing in the amplitude equation can be obtained, in principle, but must be derived for each specific system. For instance, those corresponding to the Lengyel-Epstein model [58] for the Turing instability of the CDIMA reaction have been discussed in Ref. [37]. By casting our discussion at the level of description of the amplitude equations we set the general stage for all possible generic phenomena (sufficiently close to threshold) in terms of the phase diagram of the amplitude equation parameters.

For simplicity, we restrict our analysis to systems undergoing a supercritical stationary bifurcation, at a finite critical wave-number $k_{\mathrm{c}}$ and with no intrinsic frequency, so the only possible resonance is spatial (situations where the primary instability corresponds to a Hopf bifurcation are thus excluded).

\subsection{One-dimensional amplitude equation}

The form of the amplitude equation can be derived in general by symmetry arguments. We begin with the usual separation of scales and the complex envelope $A(x, t)$ for a scalar field $A(x, t) \exp \left(\mathrm{i} k_{\mathrm{c}} x\right)+\mathrm{cc}$, where cc denotes complex conjugate, and where higher order terms are neglected. For a supercritical stationary bifurcation without forcing the amplitude equation is the Ginzburg-Landau equation with real coefficients [1]:

$$
\frac{\partial A}{\partial t}=\mu A-|A|^{2} A+\frac{\partial^{2} A}{\partial x^{2}} .
$$

We now assume that a traveling-wave modulation of a control parameter $\alpha$ of the form

$$
\alpha \rightarrow \alpha_{0}+\alpha_{1} \cos \left(k_{f} x-\omega t\right)
$$

is imposed such that the forcing wave-number $k_{f}$ is close to $n: m$ resonance with a small mismatch $q$, that is,

$$
m k_{f}=n\left(k_{\mathrm{c}}+q\right),
$$

where $m$ and $n$ are positive co-prime integers. The frequency $\omega$ is related to the velocity $v_{f}$ of the imposed modulation by the simple relation $\omega=v_{f} k_{f}$. The forcing breaks the spatial translational symmetry reflected in the phase invariance for the real Ginzburg-Landau equation, but is invariant under the combined translations $t \rightarrow t+T$ and $x \rightarrow x+\omega T / k_{f}$. The latter translation in space generates a transformation of the amplitude $A \rightarrow A \exp \left(\mathrm{i} \omega T k_{\mathrm{c}} / k_{f}\right)$. Consequently, compared to the standard amplitude equation for a supercritical bifurcation the resulting equation contains an additional invariant term:

$$
\frac{\partial A}{\partial t}=\mu A-|A|^{2} A+\epsilon\left(A^{*}\right)^{(n-1)} \mathrm{e}^{\mathrm{i}(n q x-m \omega t)}+\frac{\partial^{2} A}{\partial x^{2}} .
$$

Here the forcing strength $\epsilon$ and the distance to the (unforced) bifurcation $\mu$ are real numbers because of spatial reflection symmetry and $\epsilon$ is of order $\alpha_{1}^{m}$. The star denotes complex conjugation. For details on the derivation of the amplitude equation in the presence of small external perturbations we refer to the work of Coullet and co-workers [25,27]. The cases $n \leqslant 4$ are called strong resonances in the context of oscillatory forcing of a Hopf unstable system.

An important feature of the above non-autonomous equation is that it can be turned into an autonomous one by a simple change of variables. This corresponds to the natural choice of an amplitude, where the phase co-rotates according to the velocity $v_{f}$ of the traveling forcing. On the other hand, the explicit $x$-dependence of the new term in the amplitude equation can also be absorbed into a redefinition of the amplitude. In this way, the change of variables $A \rightarrow A \mathrm{e}^{\mathrm{i}(q x-m \omega t / n)}$ leads to the equation

$$
\frac{\partial A}{\partial t}=\left(\mu-q^{2}+\frac{\mathrm{i} \omega}{n}\right) A-|A|^{2} A+\epsilon\left(A^{*}\right)^{(n-1)}+\frac{\partial^{2} A}{\partial x^{2}}+2 \mathrm{i} q \frac{\partial A}{\partial x} .
$$

Note that the integer $m$ has been trivially removed by rescaling $m \omega$ to $\omega$. Eq. (5) is the main object of study for the case of one-dimensional patterns. It was first written for $q=0$ in Ref. [40] and in the general case in Ref. [44].

Before proceeding to analyze particular situations, let us emphasize some general features and symmetries of the equation that have important dynamical implications. Although Eq. (5) is an interesting object of study as an extended 


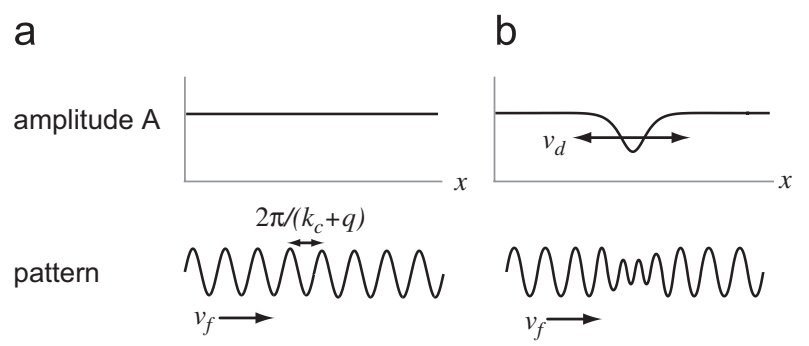

Fig. 2. Schematic plot of a homogeneous and non-homogeneous amplitude $A$ and the corresponding system's pattern.

dynamical system, it is important to keep in mind its origin for the physical interpretation of its behavior. For instance, fixed points (homogeneous and stationary solutions) of Eq. (5) correspond to periodic patterns which travel with the forcing, that is, with a periodicity $2 \pi /\left(k_{\mathrm{c}}+q\right)$ adjusted to the forcing and its velocity $v_{f}$ (Fig. 2(a)). Such solutions will be called entrained states. Traveling localized solutions of Eq. (5) imply perturbations of the original periodic pattern, which in general travel in the laboratory frame with velocities $v_{\mathrm{d}}$ different from $v_{f}$ (Fig. 2(b)).

The particular case $\omega=0$ corresponds to stationary spatial forcing, as described in [25,27,28]. Eq. (5) with $\omega=0$ has been used in the context of commensurate-incommensurate transitions in nonequilibrium systems [25], in the phase approximation and beyond. It has been shown for instance that it supports stable defects or kinks, which can be arranged in periodic or chaotic arrays [28].

The introduction of the motion of the forcing in our case, $\omega \neq 0$, is reflected by an imaginary part of the coefficient of the linear term. This is an important feature of the equation because it turns it non-gradient, that is, it can no longer be derived variationally from a Lyapunov functional. In the case $n=2$, for instance, as we will see in Section 3 , the $q=0$ problem can be mapped to that of a purely oscillatory extended system with parametric, subharmonic temporal resonance. A further interesting consequence of the non-gradient nature of the amplitude equation for $\omega \neq 0$ is that it can cause domain walls to drift $[5,44,47]$.

In this review we mostly focus on the cases $n=1$ and 2. From a general analysis of the amplitude equation, the two cases have a relevant distinction to be remarked. For the first case (1:1 resonance) the primary (stripe-forming) bifurcation of the original, unforced system becomes imperfect, in the sense of bifurcation theory, since the presence of the constant term prevents the trivial state $A=0$ from being a stationary solution. This is not the case for 2:1 resonance since, for $n=2$, the state $A=0$ is still a solution and therefore the system must undergo an instability to depart from that state.

\subsection{Two-dimensional amplitude equation}

\subsubsection{Modulations of entrained stripes}

In order to derive the right amplitude equations for $2 \mathrm{~d}$ patterns we must first fix the kind of patterns that we want to describe. In this review we will only briefly discuss the case of longitudinal and transversal modulational instabilities of basic striped patterns (see Section 5.1). From experiments in $2 \mathrm{~d}$ of the forced CDIMA reaction, stripes with longitudinal and transversal modulations have been observed to dominate the dynamics in the regime of relatively large velocities of the forcing $v_{f}$ (see Refs. $[43,45,46]$ ). With respect to the Eckhaus and zig-zag modes, in the presence of the motion of the forcing one may expect that they inherit an imaginary part in their dispersion relation, allowing for the possibility of sustained waves, both transversal and longitudinal. Experiments for large $v_{f}$ do exhibit large amplitude (in fact, nonlinear) transversal and longitudinal waves propagating in both directions (see Fig. 29) in the regime that is called "waving stripes" in Refs. [43,45]. The average position of the stripes in that mode is drifting with a very small velocity, practically at rest in the laboratory frame (this is the regime approaching the high-velocity limit of the forcing, for which the pattern has no response to the forcing and hence stands as if unforced).

Starting again with the original scaling but now including $y$-dependence we have

$$
\frac{\partial A}{\partial t}=\mu A-|A|^{2} A+\epsilon\left(A^{*}\right)^{(n-1)} \mathrm{e}^{\mathrm{i}(n q x-\omega t)}+\left(\frac{\partial}{\partial x}-\mathrm{i} \frac{\partial^{2}}{\partial y^{2}}\right)^{2} A,
$$


where the stripes are assumed to be parallel to the $y$-axis, which is appropriately scaled. As before we replace $A$ by $A^{\prime} \exp \mathrm{i}(q x-\omega t / n)$ and obtain after dropping primes

$$
\frac{\partial A}{\partial t}=\left(\mu+\frac{\mathrm{i} \omega}{n}\right) A-|A|^{2} A+\epsilon\left(A^{*}\right)^{(n-1)}+\left(\mathrm{i} q+\frac{\partial}{\partial x}-\mathrm{i} \frac{\partial^{2}}{\partial y^{2}}\right)^{2} A .
$$

Eq. (7) is the amplitude equation governing instabilities of homogeneous striped patterns. The analysis of these instabilities, which generalize the usual Eckhaus and zig-zag instabilities, will be presented in Section 5.

\subsubsection{Hexagonal symmetry at 1:1 resonance}

The most interesting aspect of two-dimensional patterns induced by traveling-stripe forcing came as a surprise from experiments of the group of Santiago de Compostela [42,45], where hexagonal patterns emerged for intermediate velocities of the forcing at 1:1 resonance in domains of parameters where those should not be expected, in principle, on the basis of the average illumination. We discuss these phenomena in Section 5.2. Here we justify the corresponding amplitude equations which will be the theoretical basis to study those phenomena. We limit the derivation of the corresponding system of amplitude equations to the case studied experimentally, that is at (nearly) exact 1:1 resonance. Patterns are thus assumed to be spatially homogeneous. This is the case in which the experimental phenomena have been understood so far. Different spatial distortion of non-homogeneous hexagonal patterns have indeed been observed in experiments, sometimes with rather intriguing behaviors [45], but a more elaborated theoretical approach is still lacking to account for those.

We describe the system as organized into stripes with a characteristic resonant wave vector along that of the forcing (complex amplitude $A$ ) in the $x$ direction, with the addition of two stripe modes (amplitudes $B_{1}$ and $B_{2}$ ) oriented at 120 degrees with respect to the forcing, so that the superposition of the three modes may potentially generate hexagons. The wave vectors associated with $A, B_{1}$, and $B_{2}$ are $\vec{k}=\hat{x} k_{\mathrm{c}}, \vec{k}_{1}=(-\hat{x}+\sqrt{3} \hat{y}) k_{\mathrm{c}} / 2$, and $\vec{k}_{2}=-(\hat{x}+\sqrt{3} \hat{y}) k_{\mathrm{c}} / 2$, respectively. The basic assumption is that the forcing has no influence on $B_{1}$ and $B_{2}$, since for those rolls the projected wave vector of the forcing is far from resonance. The amplitude equations corresponding to this situation are $[42,43]$

$$
\begin{aligned}
& \partial_{t} A=\left(\mu-g|A|^{2}-h\left(\left|B_{1}\right|^{2}+\left|B_{2}\right|^{2}\right)\right) A+\alpha B_{1}^{*} B_{2}^{*}+\epsilon \exp (-\mathrm{i} \omega t), \\
& \partial_{t} B_{1}=\left(\mu-g\left|B_{1}\right|^{2}-h\left(|A|^{2}+\left|B_{2}\right|^{2}\right)\right) B_{1}+\alpha B_{2}^{*} A^{*}, \\
& \partial_{t} B_{2}=\left(\mu-g\left|B_{2}\right|^{2}-h\left(|A|^{2}+\left|B_{1}\right|^{2}\right)\right) B_{2}+\alpha B_{1}^{*} A^{*},
\end{aligned}
$$

where we assume $h>g$, so that in the absence of the quadratic terms (i.e. for $\alpha=0$ ) stripes are preferred over hexagons.

As discussed in Section 2.1, the explicit time dependence can be eliminated by changing variables into a co-rotating frame such that $A=\bar{A} \exp (-\mathrm{i} \omega t)$ and $B_{1,2}=\bar{B}_{1,2} \exp (\mathrm{i} \omega t / 2)$ (the factor $1 / 2$ arises from the projection). Then $\mu$ is replaced in the first equation by $\mu+\mathrm{i} \omega$ and in the second and third by $\mu-\mathrm{i} \omega / 2$. Fixed points in this representation correspond to entrained solutions.

Eqs. (8-10) allow the symmetries $B_{1} \leftrightarrow B_{2},\left(B_{1}, B_{2}\right) \rightarrow\left(B_{1} \exp (\mathrm{i} \phi), B_{2} \exp (-\mathrm{i} \phi)\right)$ with an arbitrary constant phase $\phi$, which expresses translational invariance in the $y$ direction, and $\left(B_{1}, B_{2}, \alpha\right) \rightarrow\left(B_{1},-B_{2},-\alpha\right)$. The last relation expresses the fact that there are two kinds of hexagon solutions which are degenerated for $\alpha=0$. For $\alpha=0$ the stripe systems $B_{1}$ and $B_{2}$ are each fully phase invariant, i.e. independently invariant with respect to translation. We will here look for solutions with $\left|B_{1}\right|=\left|B_{2}\right|$. It is easy to verify that then the difference between the phases of $B_{1}$ and $B_{2}$ is constant in time. Accordingly, the phases can be chosen equal, i.e. $B_{1}=B_{2}(=B)$. One is then left with the symmetries $B \rightarrow-B$ and $(B, \alpha) \rightarrow(\mathrm{i} B,-\alpha)$.

The freedom to specify the scale of $A$ and $B$ allows one to choose the relevant parameters $\bar{h} \equiv h / g, \bar{\alpha} \equiv \alpha / \sqrt{g}$, $\bar{\epsilon} \equiv \sqrt{g} \epsilon$. In this representation Eqs. (8-10) take the scaled form

$$
\begin{aligned}
& \partial_{t} \bar{A}=\left(\mu+\mathrm{i} \omega-|\bar{A}|^{2}-2 \bar{h}|\bar{B}|^{2}\right) \bar{A}+\bar{\alpha} \bar{B}^{* 2}+\bar{\epsilon}, \\
& \partial_{t} \bar{B}=\left(\mu-\mathrm{i} \omega / 2-|\bar{B}|^{2}-\bar{h}\left(|\bar{A}|^{2}+|\bar{B}|^{2}\right)\right) \bar{B}+\bar{\alpha} \bar{B}^{*} \bar{A}^{*} .
\end{aligned}
$$

Note that the amplitude equations in this form correspond formally to a zero-dimensional autonomous dynamical system, since the space dependence and the explicit time dependence have been eliminated. However, since the amplitudes have a clear spatial $2 \mathrm{~d}$ interpretation, we will refer to this case as the $2 \mathrm{~d}$ case. 


\section{The autonomous dynamical system underlying resonant stripes}

The aim of this section is to provide a detailed analysis of the amplitude equation (5) in the simplest possible situation: if the solutions of this equation are spatially homogeneous. Consequently, discarding the dependences on $x$ of Eq. (5) we get

$$
\frac{\partial A}{\partial t}=\left(\tilde{\mu}+\frac{\mathrm{i} \omega}{n}\right) A-|A|^{2} A+\epsilon\left(A^{*}\right)^{(n-1)}
$$

where $\tilde{\mu}=\mu-q^{2}$. Although Eq. (13) depends originally on three parameters ( $\tilde{\mu}, \epsilon$ and $\omega$ ), a further reduction to only two parameters can be performed (see Sections 4.1 and 5.2 below). However, in this section we will keep, for clarity, all three parameters explicitly.

Eq. (13) describes generically the resonance of a stripe mode with a traveling-stripe forcing with the same orientation, regardless of the nonlinear interactions with other modes that may be present, for instance in the study of twodimensional patterns. Indeed, Eq. (13) relates to Eq. (11) for $\bar{B}=0$, and similarly it would also be present if one should study instabilities into square or rectangular patterns. Eq. (13) thus defines a (zero-dimensional) dynamical system or ordinary differential equation (ODE), which is underlying the whole class of problems with traveling-stripe resonance. In the language of dynamical systems theory, it defines a common invariant subset of the center manifolds for this class of systems, and as such it deserves a special attention.

An interesting point to remark is that Eq. (13) is a particular case of the normal form that arises if a system near a Hopf bifurcation is time-periodically perturbed close to a temporal resonance. The parameter $\omega$ in this last normal form plays the role of the frequency detuning, being proportional to the difference between a multiple of the natural frequency of the system and the frequency of the external forcing. In this way, as far as homogeneous solutions are concerned, the traveling-stripe resonance with a striped mode is exactly mapped into a problem of temporal forcing of an oscillatory system with detuning.

Although this problem is well known in the literature (see Ref. [66] for a detailed analysis), it has a central role in the present study and it is therefore important to revisit it here in detail before stepping forward into one-dimensional and two-dimensional patterns. We will thus analyze the fixed points of this equation and diverse bifurcations that may occur as these parameters are changed. We will focus mostly on the strong resonance cases $n=1$ and 2 . Other resonances will be briefly addressed in Section 3.4.

\subsection{Fixed points and bifurcations. General expressions}

Let us begin by analyzing the fixed points of Eq. (13). Recall that these fixed points correspond to patterns in the system which are entrained with the forcing.

Eq. (13) in polar coordinates $A=R \mathrm{e}^{\mathrm{i} \Theta}$ reads

$$
\begin{aligned}
& \frac{\partial R}{\partial t}=\tilde{\mu} R-R^{3}+\epsilon R^{n-1} \cos (n \Theta), \\
& R \frac{\partial \Theta}{\partial t}=\frac{\omega}{n} R-\epsilon R^{n-1} \sin (n \Theta) .
\end{aligned}
$$

The fixed points $S \mathrm{e}^{\mathrm{i} \psi}$ satisfy $\epsilon S^{n-1} \cos (n \psi)=-\tilde{\mu} S+S^{3}$ and $\epsilon S^{n-1} \sin (n \psi)=S \omega / n$. Consequently, values of $S$ are given by the roots of

$$
\left(S^{2}-\tilde{\mu}\right)^{2} S^{2}+\frac{\omega^{2}}{n^{2}} S^{2}-\epsilon^{2} S^{2(n-1)}=0,
$$

and the corresponding angles $\psi$ by

$$
\psi=\frac{1}{n} \arctan \left(\frac{\omega / n}{S^{2}-\tilde{\mu}}\right)
$$

Eq. (16) indicates that multiple fixed points, having different values of $S$, may exist. Additionally, Eq. (17) increases the multiplicity of these fixed points if $n>1$. 
Next, we study the possible (local) bifurcations of fixed points. We consider the evolution of perturbations $A=(S+$ $\delta S) \mathrm{e}^{\mathrm{i}(\psi+\delta \psi)}$. Inserting this ansatz in Eq. (13), using the conditions of the fixed points of Eqs. (16) and (17), and finally linearizing, we get the following Jacobian:

$$
\mathbf{J}=\left(\begin{array}{cc}
-(n-2) \tilde{\mu}+(n-4) S^{2} & -S \omega \\
-(n-2) \frac{\omega}{n S} & n \tilde{\mu}-n S^{2}
\end{array}\right) .
$$

Note that this Jacobian depends also on $\epsilon$ through the values of $S$ (cf. Eq. (16)). The corresponding eigenvalues are:

$$
\lambda_{ \pm}=\tilde{\mu}-2 S^{2} \pm \sqrt{\left((n-2) S^{2}-(n-1) \tilde{\mu}\right)^{2}+(n-2) \frac{\omega^{2}}{n}} .
$$

Let us now analyze this stability problem for each $n$ separately. In each case we will obtain the locations and bifurcations of the fixed points. We will find that, besides local bifurcations (saddle-node, pitchfork and Hopf), global bifurcations may also appear. In order to gain insight about these bifurcations, we will typically visualize them in two different parameter spaces. In both cases the forcing velocity $\omega$ will be one parameter and the second will be either the strength of the forcing $\epsilon$ or the distance to the instability threshold of the unforced system $\tilde{\mu}$. Note also that both parameter spaces are symmetric with respect to $\omega$, since only the square of its value enters in Eqs. $(16,19)$. In the $(\epsilon, \omega)$ space the region where the entrainment occurs will get smaller as $\epsilon$ decreases. This horn shaped area is known as Arnol'd tongue [3].

\section{2. $1: 1$ resonance}

For the case $n=1$, Eq. (16) becomes

$$
\left(S^{2}-\tilde{\mu}\right)^{2} S^{2}+\omega^{2} S^{2}=\epsilon^{2}
$$

with $\psi=\arctan \left(\omega /\left(S^{2}-\tilde{\mu}\right)\right)$. Since Eq. (20) is cubic in $S^{2}$, either one or three solutions for $S^{2}$ exist. This information is provided by the discriminant of the equation $D_{1}=-27 \epsilon^{4}+4\left(\tilde{\mu}^{3}+9 \omega^{2} \tilde{\mu}\right) \epsilon^{2}-4 \omega^{2}\left(\tilde{\mu}^{2}+\omega^{2}\right)^{2}$. If $D_{1}>0\left(D_{1}<0\right)$, three (respectively, one) real values exist. These fixed points are generated and destroyed pairwise by saddle-node bifurcations.

The region where three fixed points exist is indicated with light gray in Fig. 3, whereas in the rest of the parameter space only one fixed point exists. The region of multistability ends in a cusp at the critical point $\omega=\tilde{\mu} / \sqrt{3}, \epsilon=\sqrt{8 \tilde{\mu}^{3} / 27}$ where Eq. (20) has 3 identical solutions with $S^{2}=2 \tilde{\mu} / 3$.

When the three fixed points are present, they have different stability properties. One is stable and the other two are unstable with one of them being of saddle point and the other a repellor. Both unstable fixed points disappear through a saddle-node bifurcation at the dotted line in Fig. 3. On the solid lines the stable and the saddle fixed points also disappear through a saddle-node bifurcation. A closer analysis shows that this bifurcation is of global type, since a limit cycle appears after both fixed points collide. This type of bifurcation is usually called SNIPER (Saddle-Node Infinite PERiod bifurcation on a limit cycle, [67]).

We can now study the stability of these fixed points. The eigenvalues are given by $\lambda_{ \pm}=\tilde{\mu}-2 S^{2} \pm \sqrt{S^{4}-\omega^{2}}$. If $S^{2}$ becomes smaller than $|\omega|$ and $\tilde{\mu} / 2$ (the latter condition is equivalent to $\epsilon<\sqrt{\tilde{\mu}^{3} / 8+\tilde{\mu} \omega^{2} / 2}$ ), a Hopf bifurcation occurs with frequency

$$
\Omega_{\text {Hopf }}=\sqrt{\omega^{2}-\tilde{\mu}^{2} / 4}=\sqrt{2 \epsilon^{2} / \tilde{\mu}-\tilde{\mu}^{2} / 2} .
$$

This bifurcation curve is indicated with a dashed line in Figs. 3(a) and (b). This curve divides the region where only one fixed point exists, into two parts. In this figure we also sketch some typical orbits of these three different possible situations.

Near the critical point where the region of multiple solutions ends in a cusp, the dynamics of Eq. (13) is more complex. This region is shown in Fig. 4(a). The Hopf bifurcation also occurs inside the multistability region. Consequently, two small regions of bistability exist. In one case this bistability is between two fixed points and in the other between a fixed point and a limit cycle. This small limit cycle disappears through a homoclinic bifurcation at the curve indicated by a dot-dashed line in Fig. 4.

Another interesting issue concerning the 1:1 resonance case is that inside the region where a stable limit cycle exists (the white regions in Figs. 3(a), (b) and 4(a)), we can distinguish two different situations. In the first case the 
a

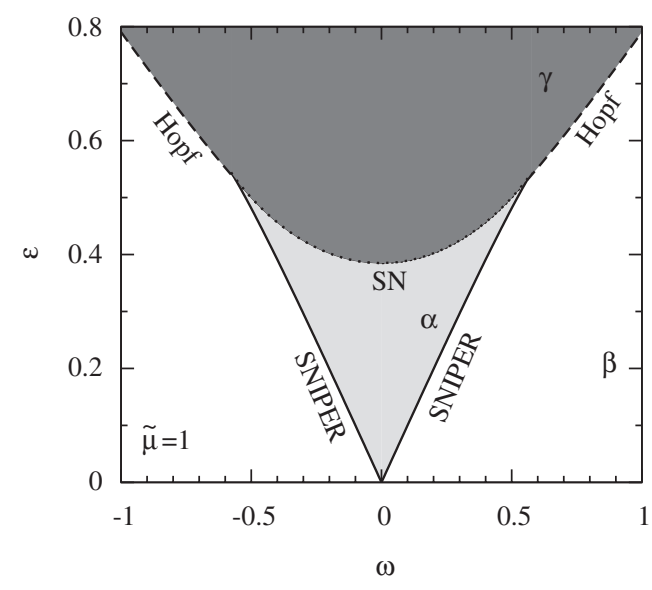

$(\alpha)$

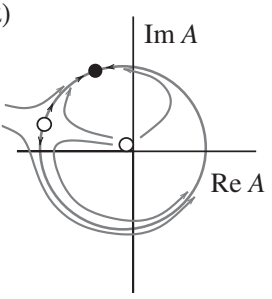

b
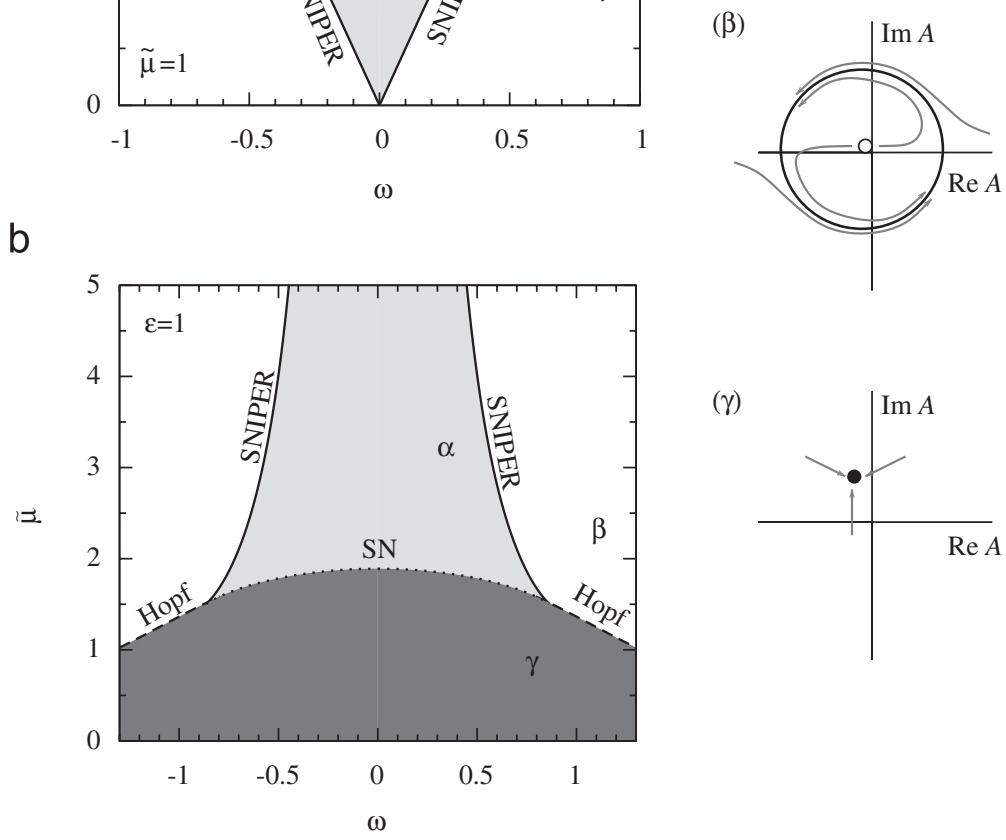

$(\gamma)$

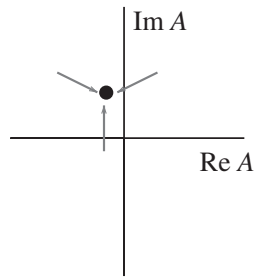

Fig. 3. Entrainment region in the parameter spaces $\epsilon$ vs. $\omega$ in (a) and $\tilde{\mu}$ vs. $\omega$ in (b) for the $1: 1$ resonance case (with $\tilde{\mu}=1$ and, respectively, $\epsilon=1$ ). The parameter space $\epsilon$ vs. $\omega$ in (a) corresponds to keeping constant distance to the bifurcation of the unforced system and varying the amplitude of the forcing $\epsilon$ and its frequency $\omega$. The Arnol'd tongue is shown in gray. Three fixed points exist in the light gray area. Outside this region only one fixed point exist. This fixed point is stable in the dark gray area. It becomes unstable through a Hopf bifurcation at the dashed-line. The full lines indicate the location of SNIPER bifurcations. The dotted line (denoted by SN) indicates the saddle-node bifurcation involving the unstable and saddle-node fixed points. In $(\alpha),(\beta)$ and $(\gamma)$ we sketch three possible behaviors in the complex $A$ place (see also Fig. 4).

amplitude of the limit cycle is small, and consequently it does not encircle the origin $A=0$ whereas in the second it does (cf. diagrams $(\chi)$ and $(\eta)$ in Fig. 4). This difference is of relevance for the entrainment of the pattern to the external forcing. The transition corresponds to a change between a frequency-locked state and a non-synchronous case [3]. Since in the first case the average velocity of the corresponding wave in the original system coincides with that of the forcing one can include such states into the entrained regime. In this review we will use "entrained" for the fixed-point states, whereas modulated but locked states will be called "entrained oscillatory". The word "oscillatory" will usually refer to modulated waves that are locked to the forcing and those that are not.

\section{3. $2: 1$ resonance}

In the 2:1 resonance case the origin, $S=0$, is always a solution of Eq. (13). Consequently, Eq. (16) reduces to a quadratic equation. $S$ can assume one or two further values with $S \neq 0$ given by

$$
S^{2}=\tilde{\mu} \pm \sqrt{\epsilon^{2}-\frac{\omega^{2}}{4}} .
$$



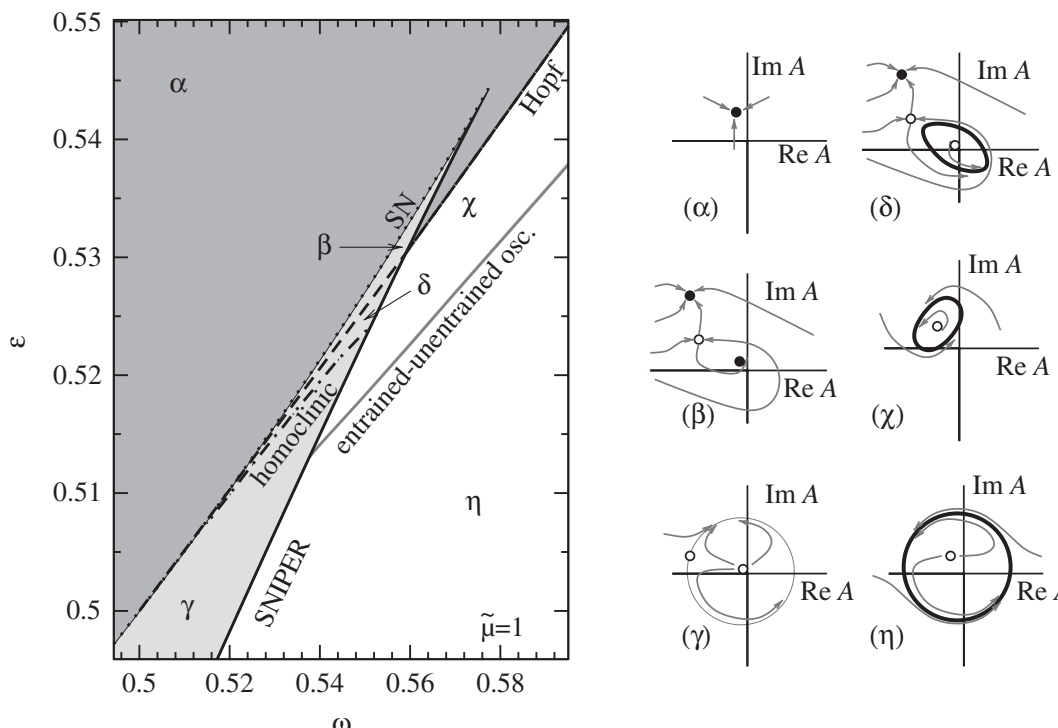

$(\delta)$
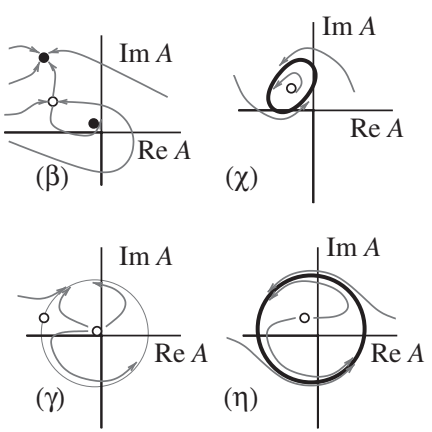

Fig. 4. The left panel shows a magnification of the region near the critical point of the 1:1 resonance (where the three fixed points end in a cusp) where complex dynamics occur (with $\tilde{\mu}=1$ as in Fig. 3(a)). This critical point is located at $\epsilon=\sqrt{8 / 27}$ and $\omega=\sqrt{1 / 3}$. In $(\alpha)$ to $(\eta)$ sketches of the possible dynamics are shown. Note, in particular, the existence of bistability between two stable fixed points (in $(\beta)$ ) and between a stable fixed point and a stable limit cycle (in $(\delta)$ ). The dashed line inside the dark gray region indicates the transition between these two behaviors. The last possibility disappears when the small limit cycle collides with the intermediate saddle-node point. The location of this homoclinic-loop bifurcation is indicated with a dot-dashed line. The gray full line indicates the parameters values where the limit cycle touches $R=0$ (note that this qualitative change is not a bifurcation).

Eq. (17) provides two possible angles for each $S \neq 0$. Thus we may have either one, three or five fixed points. In Figs. 5(a) and (b), the regions where at least one stable fixed point exists are indicated with gray. Here we can distinguish between three different cases, depending on the number of fixed points.

If five fixed points exist (light gray in Fig. 5) then two are stable with coordinates $A_{ \pm}=S_{ \pm}$exp i $\psi_{ \pm}$, with $S_{ \pm}^{2}=\tilde{\mu}+$ $\sqrt{\epsilon^{2}-\omega^{2} / 4}$ and angles $\psi_{+}=\frac{1}{2} \arctan \left(\omega / \sqrt{4 \epsilon^{2}-\omega^{2}}\right)$ and $\psi_{-}=\pi+\frac{1}{2} \arctan \left(\omega / \sqrt{4 \epsilon^{2}-\omega^{2}}\right)$. The remaining fixed points are unstable, two of them being saddle fixed points (cf. Fig. $5(\alpha)$ ). Both saddle points disappear pairwise with the stable $A_{ \pm}$through saddle-node bifurcations at $|\epsilon|=|\omega / 2|$. As we already saw for the $1: 1$ case, this bifurcation is global and corresponds to two simultaneous SNIPER bifurcations which give rise to a limit cycle (see sketches $(\alpha)$ to $(\delta)$ in Fig. 5). Additionally, both saddle fixed points disappear via a pitchfork bifurcation involving the unstable fixed point at $S=0$ when $\tilde{\mu}^{2}=\epsilon^{2}-\omega^{2} / 4$. This bifurcation is indicated with a dotted line denoted PF1 in Figs. 5(a) and (b). A further pitchfork bifurcation (PF2) between two stable and one unstable fixed point occurs for negative values of $\tilde{\mu}$ (indicated with a dot-dashed line in Figs. 5(a) and (b)).

The linear stability analysis of the fixed point $S$ is very simple. We obtain that a Hopf bifurcation occurs for $\tilde{\mu}=0$ (and $|\omega / 2|>1$ ) as indicated by the dashed lines in Fig. 5(b). The Hopf bifurcation together with the PF2 pitchfork bifurcation corresponds to the original instability in the unforced case.

\subsection{Higher order resonances}

In this review we will not address in detail the resonances of higher order $(n>2)$, since the analysis is qualitatively similar (see e.g. [66]). Typically, one distinguishes between strong and weak resonances. In our case the borderline order is $n=4$, for which the resonant term $\epsilon\left(A^{*}\right)^{(n-1)}$ of the amplitude equation (5) is a nonlinearity of the same order than the lowest nonlinearity of the unforced equation $(\epsilon=0)$. Weak resonances will be those for which the resonant term is of nonlinear order higher than cubic. The analysis of the remaining strong resonances $(n=3,4)$, and also the weak resonances for $n>4$, is very similar to the case $n=2$. Generally, a wedge shaped region in the space $\epsilon$ vs. $\omega$ is observed, where $n$ different stable fixed points exist. The limits of this Arnol'd tongue are given by $n$ simultaneous SNIPER bifurcations. Outside the tongue only a stable limit cycle exists (for $\tilde{\mu}>0$ ). 
a

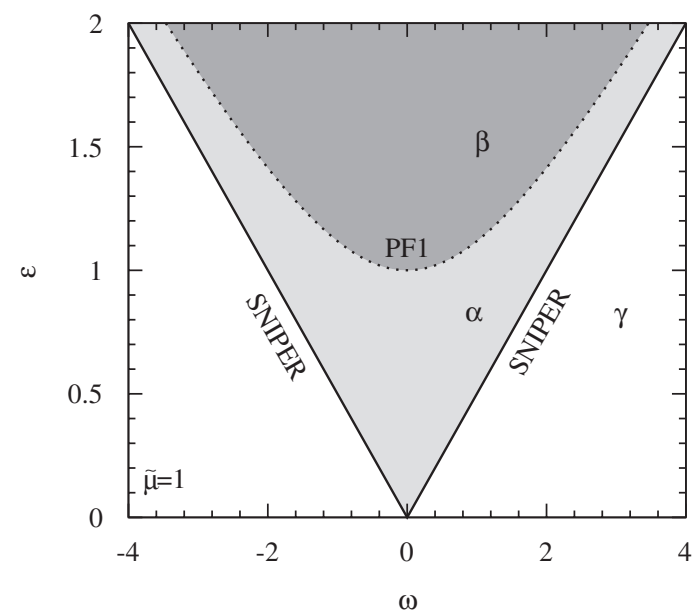

$(\alpha)$

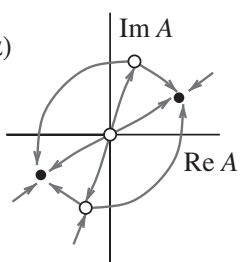

$(\beta)$

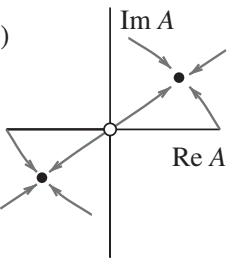

b

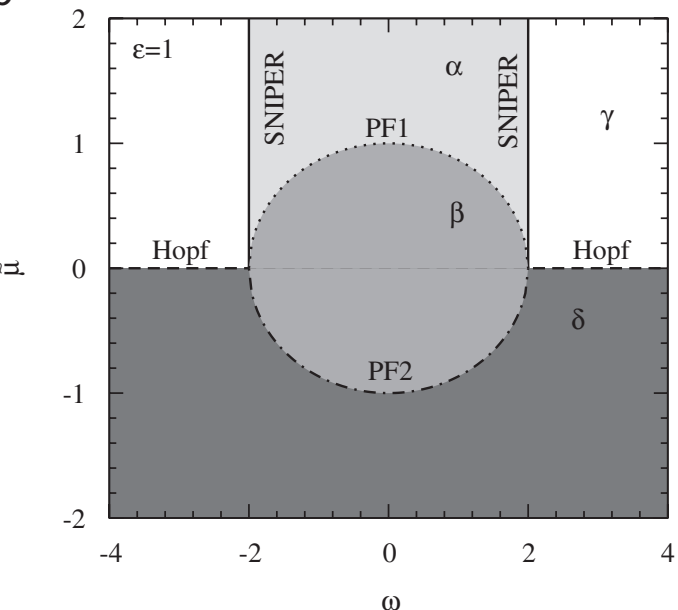

$(\gamma)$

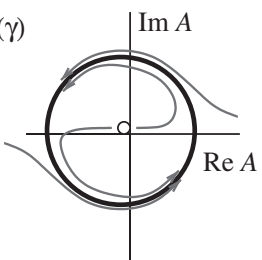

( $)$

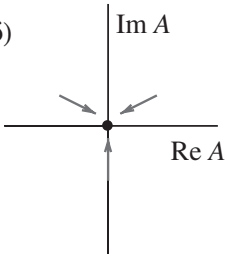

Fig. 5. Entrainment region for the 2:1 resonance. In (a) $\tilde{\mu}$ is kept constant to 1, this corresponds to keeping constant distance to the bifurcation of the unforced system and varying the amplitude of the forcing $\epsilon$ and its frequency $\omega$. In (b) $\epsilon=1$. In the light gray region five fixed points exist, two of them are stable. In the mid gray region three exist where also two are stable. In the rest of the phase space a unique fixed point exists. It is stable in the dark gray region and in the white region a stable limit cycle exists. The location where this limit cycle is generated by a Hopf bifurcation is indicated by a dashed line. This limit cycle disappears through a SNIPER bifurcation (full lines).

\section{One-dimensional patterns. Localized solutions}

In this section we consider non-homogeneous solutions of amplitude equation (5) in one spatial dimension. Homogeneous solutions of the amplitude equation correspond to spatially periodic patterns of the original system, therefore non-homogeneous solutions imply some kind of distortion of the underlying periodicity. We can distinguish three basic mechanisms by which a non-homogeneous solution can emerge. The first mechanism is an instability of the homogeneous solution with respect to small periodic perturbations. This is a generalization of the usual Eckhaus and zig-zag instabilities, which will be discussed in Section 5.1.

Further, one can find defects that connect spatial domains where the solution is homogeneous. Often, deterministic forcing is used to remove defects and to obtain ideal patterns. Here, however, we find that the forcing can support defects between regions of stripes that have different phases with respect to the stripes of the forcing. Below we describe existence and stability of these defect states. Since solitary defects correspond to homoclinic or heteroclinic solutions of spatial ODEs we can use boundary value solvers and numerical continuation to find the existence range of 
defects. Furthermore, stability and existence of such solutions can also be studied by direct numerical simulations of the partial differential equation. This analysis will be the main focus of this section.

As we will see below, the defects typically travel, which can be attributed to the breaking of all spatial reflection symmetries. The velocity of this drift depends non-trivially on the various parameters, such as $\mu, \epsilon, \omega, q$, and also the distance $d$ between defects. The velocity can in general be obtained only from numerical solutions. However, for large $\mu$ one can reduce the dynamics of the amplitude equation to that of a phase equation and then, in certain cases, derive analytical approximations of the drift velocity.

A third type of non-homogeneous patterns is due to the transition to time dependence in the homogeneous problem if $\omega$ is increased, typically through the SNIPER bifurcation. The solution can then be either homogeneous and time dependent (i.e. the solution studied in Section 3) or non-homogeneous. For values of $\omega$ that are beyond but close to the SNIPER, one observes that non-homogeneous solutions consist of localized structures which are similar to the defects. A few observations on these solutions are therefore made when we discuss defects in this section.

\subsection{Traveling pulses and kinks in the amplitude equation}

To simplify the discussion of domain walls in Eq. (5) we can, if $n \neq 4$, re-scale time, space and $A$ to reduce the number of parameters. Here we choose to scale the forcing strength to 1 and introduce $\hat{t}=\epsilon^{2 /(4-n)} t, \hat{x}=\epsilon^{1 /(4-n)} x$, $\hat{A}=\epsilon^{1 /(n-4)} A, \hat{\mu}=\epsilon^{2 /(n-4)} \mu, \hat{q}=\epsilon^{1 /(n-4)} q$, and $\hat{\omega}=\epsilon^{2 /(n-4)} \omega$. With these rescalings and after removing hats Eq. (5) reads

$$
\frac{\partial A}{\partial t}=\left(\mu-q^{2}+\frac{\mathrm{i} \omega}{n}\right) A-|A|^{2} A+\left(A^{*}\right)^{(n-1)}+\frac{\partial^{2} A}{\partial x^{2}}+2 \mathrm{i} q \frac{\partial A}{\partial x} .
$$

An useful representation of $A=R \operatorname{exp~} \mathrm{i} \Theta$ in terms of its modulus $R$ and phase $\Theta$ results in:

$$
\begin{aligned}
& \frac{\partial R}{\partial t}=\tilde{\mu} R-R^{3}+R^{n-1} \cos n \Theta+\frac{\partial^{2} R}{\partial x^{2}}-R\left(\frac{\partial \Theta}{\partial x}\right)^{2}-2 q R \frac{\partial \Theta}{\partial x}, \\
& \frac{\partial \Theta}{\partial t}=\frac{\omega}{n}-R^{n-2} \sin n \Theta+\frac{\partial^{2} \Theta}{\partial x^{2}}+\frac{2}{R} \frac{\partial R}{\partial x} \frac{\partial \Theta}{\partial x}+\frac{2 q}{R} \frac{\partial R}{\partial x},
\end{aligned}
$$

where we have introduced again $\tilde{\mu}=\mu-q^{2}$.

\subsubsection{Spatial ODEs}

Defect solutions of Eq. (23) can be obtained by fixing its time dependence and regarding it as an ODE with a new "time" variable $x$. To this end we assume $A(x, t)=A(x-v t)$ and get

$$
0=\left(\tilde{\mu}+\frac{\mathrm{i} \omega}{n}\right) A-|A|^{2} A+\bar{A}^{n-1}+\partial_{x}^{2} A+(2 \mathrm{i} q+v) \partial_{x} A .
$$

We then write $A=X+\mathrm{i} Y$ and obtain, after introducing further variables $U$ and $V$,

$$
\begin{aligned}
& \partial_{x} X=U, \\
& \partial_{x} Y=V, \\
& \partial_{x} U=\frac{\omega}{n} Y-\tilde{\mu} X+\left(X^{2}+Y^{2}\right) X-\operatorname{Re} F+2 q V-v U, \\
& \partial_{x} V=-\frac{\omega}{n} X-\tilde{\mu} Y+\left(X^{2}+Y^{2}\right) Y-\operatorname{Im} F-2 q U-v V,
\end{aligned}
$$

where

$$
F=(X-\mathrm{i} Y)^{n-1},
$$

and Re and Im denote the real and imaginary part, respectively. The fixed point solutions of these equations are the fixed points obtained in Section 3 (with $\epsilon=1$ ) together with $U=V=0$. 
a

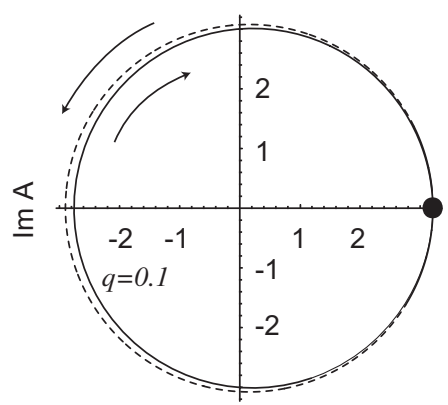

$\operatorname{Re} A$ b

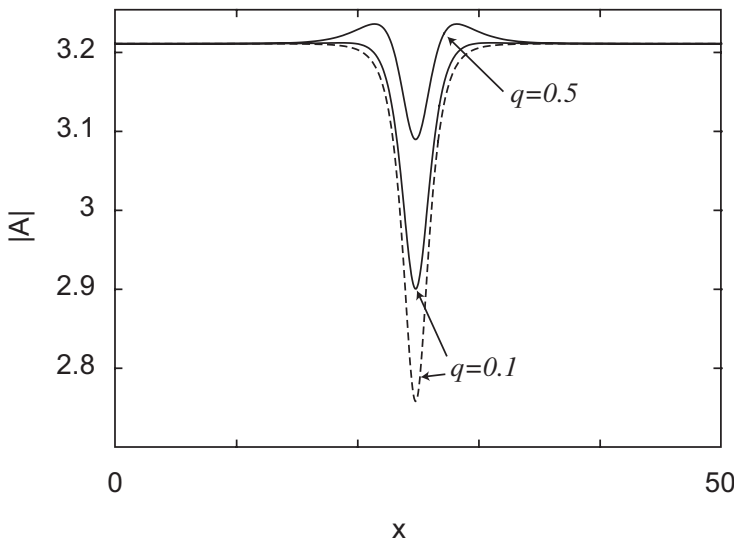

Fig. 6. Real and imaginary parts (a) and the modulus $|A|$ vs. $x$ (b) of pulse solutions in $1: 1$ resonance for $\tilde{\mu}=10, \omega=0$. Pulses can be either of clockwise chirality (solid curves) or counterclockwise chirality (dashed curves). In (a) the curves are projections of pulses that approach the homogeneous fixed point solution (large dot) in the limit of $x=-\infty$ and $\infty$. The projections of clockwise and counterclockwise pulses differ for non-zero $q$.

A further possibility consists in writing the spatial ODE in terms of phase and modulus of the amplitude $A$ as introduced in $(24,25)$. This system of equations has been used for the analysis of the case $n=1$ described in Section 4.1.2, and will be regarded as equivalent in the following discussions.

Defects are found as homoclinic or heteroclinic connections in the spatial ODEs (27-30). We have used numerical continuation by the HOMCONT routine of XPPAUT to trace the corresponding defect solutions with parameters $\tilde{\mu}, q$ and $\omega$. HOMCONT calculates homoclinic and heteroclinic orbits by approximating them by orbits of a given interval in time (here the space coordinate $x$ ). For details on the numerical procedures we refer to Refs. $[68,69]$.

\subsubsection{Case $n=1$. Pulses}

Defects generated by the 1:1 resonance are kinks, which travel unless $\omega=0$. They are localized perturbations of the pattern, where the phase rotates by $2 \pi$ and, depending on the parameters, a small or strong distortion of the modulus occurs (see Fig. 6). They connect spatial domains, that are locked to the forcing. Since the domains on both sides are equivalent, the kinks exhibit similarities to pulses in excitable media. Therefore they will be called pulses in the following. We also note that in general there are two kinds of pulses: those that decrease the phase $\Theta$ with increasing spatial coordinate $x$ (clockwise chirality, see Fig. 6(a)) and those increasing it (counterclockwise chirality). As will be discussed below, the symmetry between the two pulses is broken for $q \neq 0$, such that one chirality is preferred by the system.

Pulses exist if the forcing is weak or if the control parameter is large. For the scaling used in this section ( $\epsilon$ scaled to 1) both conditions correspond to a large $\mu$. In the limit of large $\mu$ one can apply the phase approximation (effectively ignoring the spatial mismatch $q$ ) and derive expressions for the existence range and velocity of pulses. This approach will be discussed in Section 4.2.

In this section we will briefly describe the limits of existence of pulses for intermediate values of $\tilde{\mu}$ where numerical analysis is necessary. The case $q=0$ has been addressed already in the literature in the context of oscillatory media $[15,16]$. Because of spatial reflection symmetry for $q=0$ the two pulses of clockwise and counterclockwise chirality are equivalent. For decreased $\tilde{\mu}$ they disappear in a saddle-node bifurcation, where a stable pulse solution merges with an unstable one (Fig. 7, the pulse saddle node is indicated by a dashed curve). For $\omega=0$ this occurs by decreasing $\tilde{\mu}$ through $\tilde{\mu} \approx 7.22$. Calculations with the HOMCONT method and direct numerical simulations of Eq. (23) showed that the critical $\tilde{\mu}$ value is shifted to smaller values with increased $|\omega|$ (Fig. 7).

The second special case we want to discuss is that of a non-zero $q$ for $\omega=0$. An important difference is that now we need to distinguish the two pulses according to their chirality. Pulses of clockwise and counterclockwise chirality are shown in Fig. 6. Depending on the sign of $q$, one or the other pulse is preferred, or more precisely, the range of 


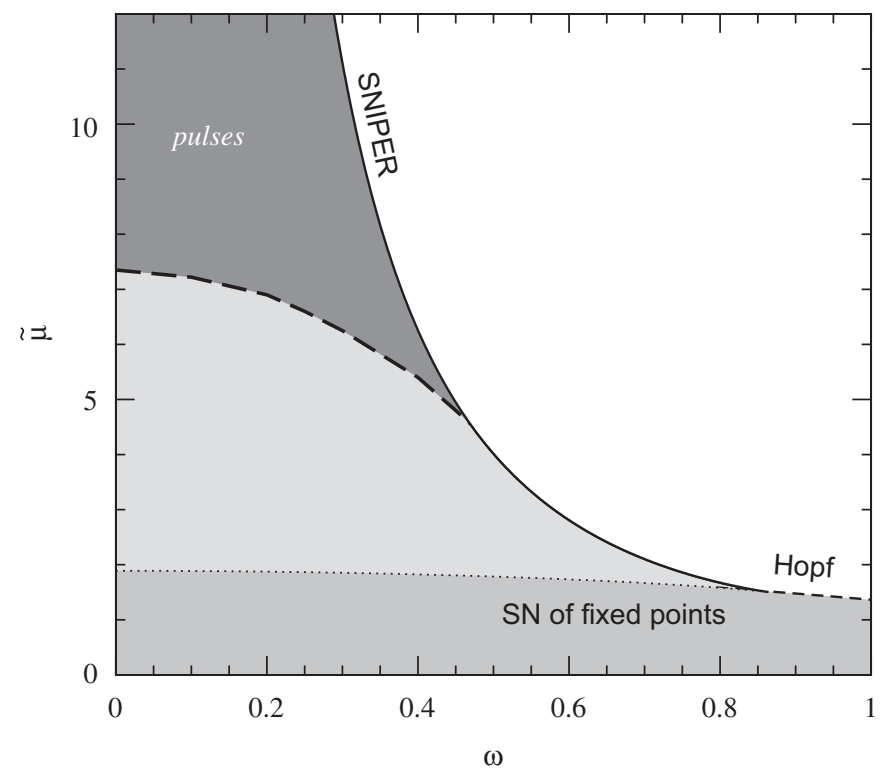

Fig. 7. Region of existence of pulses in the 1:1 case with $q=0$. The boundary was calculated by direct numerical integration of Eq. (23). Pulses are only stable inside the dark-gray region. The stable pulses disappear at the thick dashed line (they annihilate with a second family of unstable pulses in a saddle-node bifurcation).

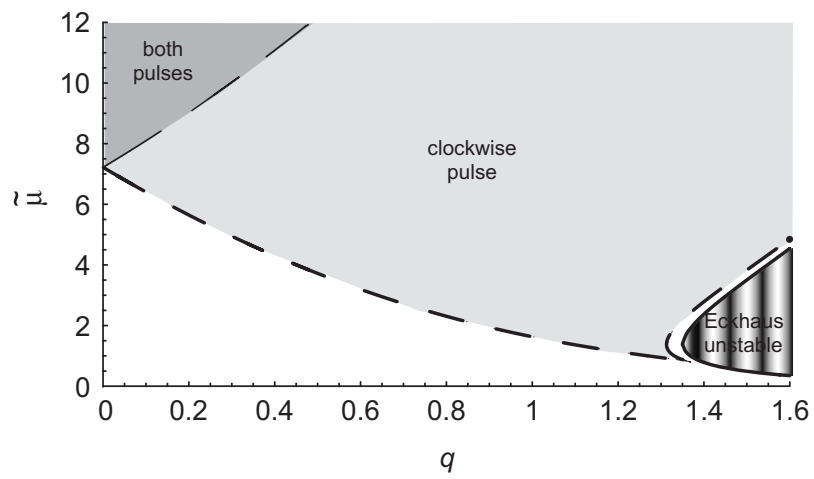

Fig. 8. Limits of existence of solitary pulse solutions (dashed lines) and generalized Eckhaus instability (solid line) for $n=1$ and $\omega=0$. The entrained state is unstable against periodic modulations to the right of the Eckhaus line. Pulses with clockwise and counterclockwise chirality exist above the thick and thin dashed line, respectively. The thick dashed line exhibits a cusp point close to the Eckhaus instability.

existence of one of the pulses becomes larger. This property is related to the fact that a nonzero $q$ introduces a mismatch and the system uses pulses to accommodate both the wave number of the forcing and that of the internal instability by introducing $2 \pi$ shifts in the phase in the appropriate direction.

The range of existence of pulses in the $\omega=0$ case is shown in Fig. 8. Pulses decreasing (clockwise chirality) or increasing (counterclockwise chirality) the phase exist above the thick and thin dashed line, respectively. With increasing $q$ the existence range of the clockwise pulse increases strongly while the range of the second pulse decreases. Intuitively, it can be understood that phase-decreasing clockwise pulses are preferred by the pattern for $q>0$, since the wavenumber $k_{f}=k_{\mathrm{c}}+q$ of a pattern without pulses is larger than the pattern forming wave-number $k_{\mathrm{c}}$ and a decrease of the phase results in a decrease of the "effective" wave-number.

The mechanism by which pulses disappear at the dashed lines in Fig. 8 is a saddle-node bifurcation, where the stable pulse solution collides with an unstable one. The cusp exhibited by the thick dashed line in Fig. 8 at $q \approx 1.4$ appears 


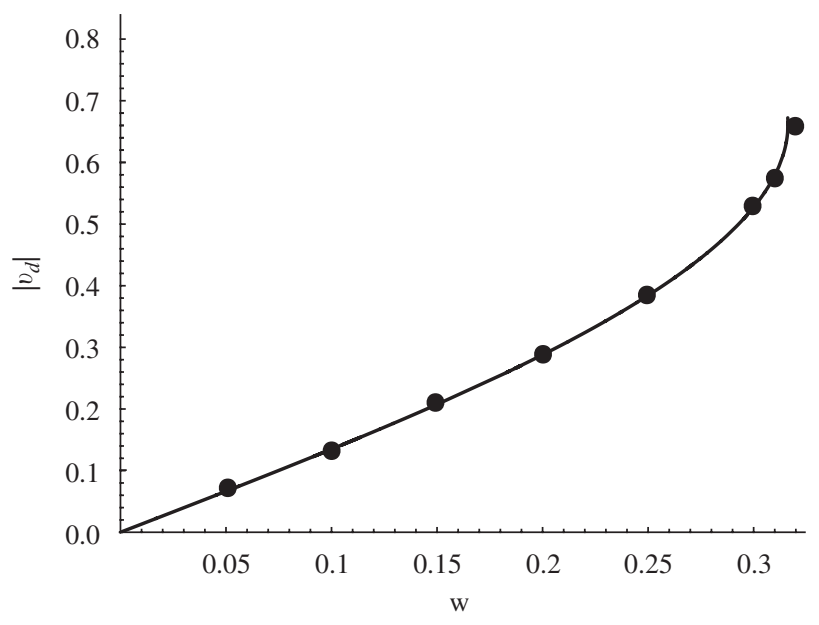

Fig. 9. The velocity of pulses for $n=1, q=0$, and $\tilde{\mu}=10$. Dots are numerical results from simulations of Eq. (23) in a periodic domain of length 50. The solid line is obtained by numerical continuation using HOMCONT.

to be related to the modulational instability (solid line). This instability generalizes the usual Eckhaus instability of striped patterns to the case of forced patterns and was first calculated by Coullet and Repaux [27] in the case of $\omega=0$ (see Section 5.1). Numerical simulations of the amplitude equation have shown that by crossing the curve of pulse existence in this region of the parameter space (dashed line close to the Eckhaus) the system jumps to a periodic state with a well-defined wave number. The origin of the slight shift between the Eckhaus and the pulse saddle-node line remains unclear.

Next we discuss the propagation of pulses. All pulses for $\omega \neq 0$ travel. For intermediate $\mu$ values the propagation velocity can only be determined numerically. Fig. 9 shows the velocity of a pulse for $q=0$ and $\tilde{\mu}=10$. The velocity depends linearly on $\omega$ for small velocities, but becomes strongly non-linear for larger $\omega$. Similar behavior was found for a large range of $\mu$ and $q$ parameters and will be studied in Section 4.2 using the phase approximation. At $\omega \approx 0.32$ the stable pulse collides with an unstable pulse in a saddle-node and, at the same time, the fixed point solution disappears in a SNIPER (cf. Fig. 7).

We finally mention that pulse interaction was studied in the case of $\omega=0$ in [28]. Interaction of sufficiently distant defects is governed by the eigenvalues of homogeneous fixed point solutions of Eqs. (27-30). In [28] a transition was found, that turns interaction of two adjacent defects, which is monotonic with the distance of the defects, to one where the mutual attraction/repulsion oscillates with distance. It is a well-known application of dynamical systems theory that for oscillating defect interaction (or indeed for complex spatial eigenvalues) spatially chaotic arrays of pulses can be stable $[1,28,29]$. The generalization of this transition to the case $\omega \neq 0$ has not been studied systematically for the case $n=1$ but was recently studied for the case $n=2$ [47]. In the following section a few results will be discussed.

\subsubsection{Case $n=2$. Kinks}

Defects in 2:1 resonance connect the two stable fixed points $A_{ \pm}$given in Section 3.3, which exist if $|\omega|<2$. The phase in these defects, called kinks, rotates by $\pi$. In contrast to the 1:1 case, stable defects exist for all parameters values where non-trivial fixed points exist, that is, in regions $\alpha$ and $\beta$ in Fig. 5 (except for possible instabilities of kinks for large $q$ ).

It has been known for some time that for $q=0$ one can distinguish two qualitatively different types of kinks [5]. For small $\mu$ one finds Ising kinks whose real and imaginary parts pass through zero simultaneously, such that the amplitude vanishes at this point. There is one Ising kink connecting the two fixed points in each direction (Fig. 10(a)). For Bloch kinks the real and imaginary parts pass through zero at different locations. There are two Bloch kinks connecting the fixed points in each direction (Fig. 10(b)). 
a

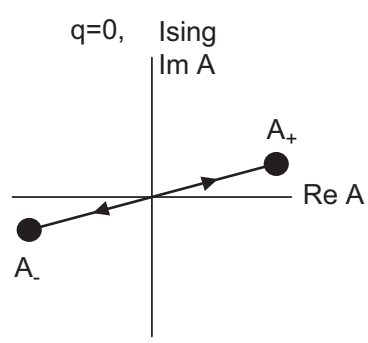

b

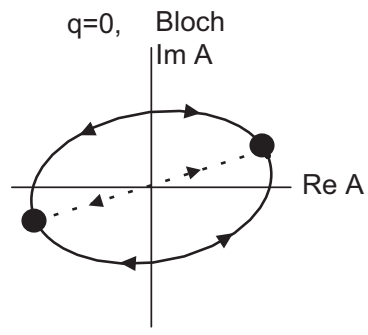

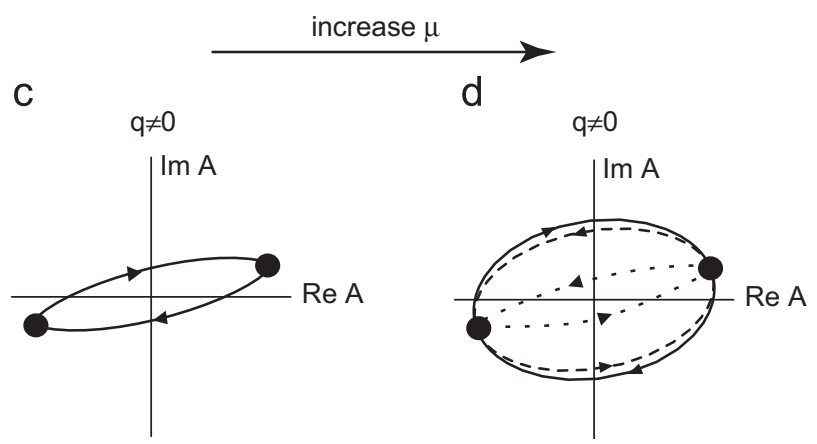

Fig. 10. Schematic plot of domain walls in the complex space of $A$ as they occur in Eq. (23) for $n=2$. The curves display the connections between fixed points (large dots) that are approached in the limit of $x=-\infty$ and $\infty$. These fixed points move away from the real axis with increasing $\omega$ (see Section 3.3). Dashed and dotted connections in (d) correspond to walls that are created in the saddle-node of the imperfect NIB transition.

For $\omega=q=0$, Ising and Bloch kinks of Eq. (23) can be expressed analytically in terms of real and imaginary parts of $A=X+\mathrm{i} Y[5]$ :

$$
\begin{aligned}
& X_{\mathrm{I}}= \pm \sqrt{\mu+1} \tanh (\sqrt{(\mu+1) / 2} x), \quad Y_{\mathrm{I}}=0, \\
& X_{\mathrm{B}}= \pm \sqrt{\mu+1} \tanh (\sqrt{2} x), \quad Y_{\mathrm{B}}= \pm \sqrt{\mu-3} / \cosh (\sqrt{2} x),
\end{aligned}
$$

where we have assumed that the kink is situated at $x=0$.

For $|\omega|<2$, a pitchfork bifurcation between both kink types occurs at a critical $\tilde{\mu}_{\mathrm{c}}(\omega)=\sqrt{9-9 \omega^{2} / 4}[70]$. The bifurcation is illustrated in Fig. 11 (dashed lines) and is called nonequilibrium Ising-Bloch (NIB) transition. Owing to the non-gradient term in (23), Bloch kinks drift for $\omega \neq 0[5]$.

Next, we will discuss the consequences of $q \neq 0$. A non-zero $q$ acts as a perturbation of the Ising and Bloch kinks and renders the NIB bifurcation imperfect. One can then find for small $\tilde{\mu}$ two stable kinks with relatively small departure from an Ising solution (Fig. 10(c)). The kink connecting $A_{+}$with $A_{-}$is traced in Fig. 11 and denoted by B1. Increasing $\tilde{\mu}$ through a turning point of the imperfect bifurcation, four stable kinks (B1 and B2) and two unstable kinks (I) exist (see Figs. 10(d) and 11). Note that a non-zero $q$ imposes a chirality for all kinks. Similar to the 1:1 case, with increasing $q$ the system prefers one of the kinks, that is, the second kink ( $B 2$ in Fig. 11) is shifted to higher $\tilde{\mu}$-values.

All walls, except Ising walls for $q=0$, drift provided that $\omega$ is non-zero. The following results on drift velocities were obtained by numerical integration of Eq. (23) in a spatially periodic domain with two kinks. An example of the basic setup is shown in Fig. 12. It is worth noting that kink evolution in these simulations is not affected by interaction since they are relatively far from each other. Interaction of kinks is usually exponentially decaying with their distance $d$ and thus for large distances the kink distance remains effectively constant. Therefore interaction of kinks does not disturb the measurements of kink velocities in these simulation.

Fig. 13 shows the drift velocity $v_{\mathrm{d}}$ for different choices of the domain length $L=2 d$. For small values of $\omega$ a linear relation between the forcing velocity and the velocity of the kinks is apparent. For $\omega$ values close to 2, i.e. close to the SNIPER bifurcation of fixed points, the linear relation is lost. This behavior is qualitatively similar to the $1: 1$ case. As $\omega$ approaches the critical point the velocity grows more strongly and reaches a value of approximately 1.687 at $\omega=2$ in the limit of large $L$. 


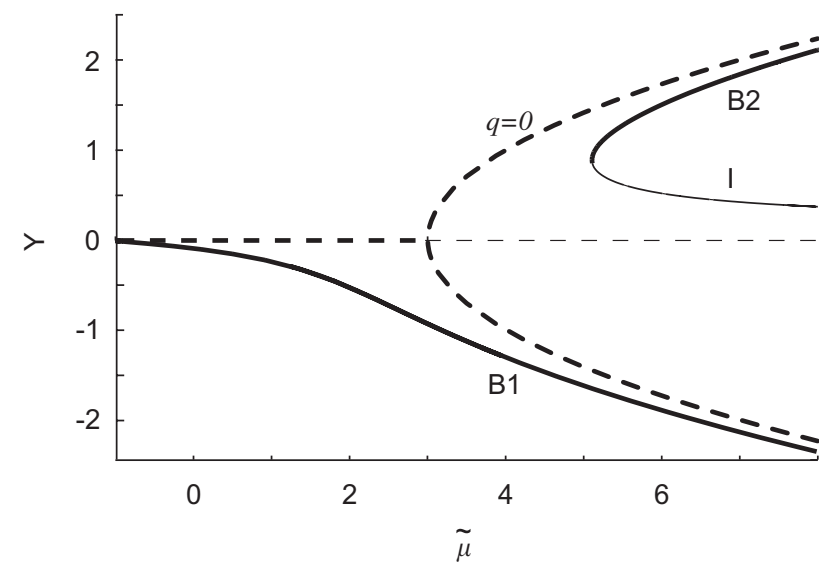

Fig. 11. The NIB transition for $q=0$ (dashed lines) and the imperfect transition for $q=0.1$ (solid lines). In both cases $\omega=0$ so that the imaginary part $Y$ of fixed points $A_{ \pm}$is zero. The graphs show the $Y$-value at the center of each kink connecting $A_{+}$with $A_{-}$. The two stable branches (denoted B1, B2) correspond to one pair of solid and dashed connections in Fig. 10(d) (although there $\omega \neq 0$ ).

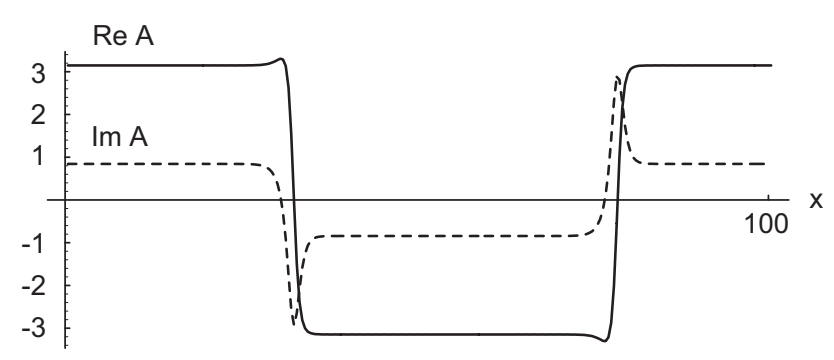

Fig. 12. A solution of Eq. (23) for $n=2$ with two kinks ( $\mu=10, q=0.5, \omega=1$ ). We have plotted the real (solid) and imaginary (dashed) part of $A$.

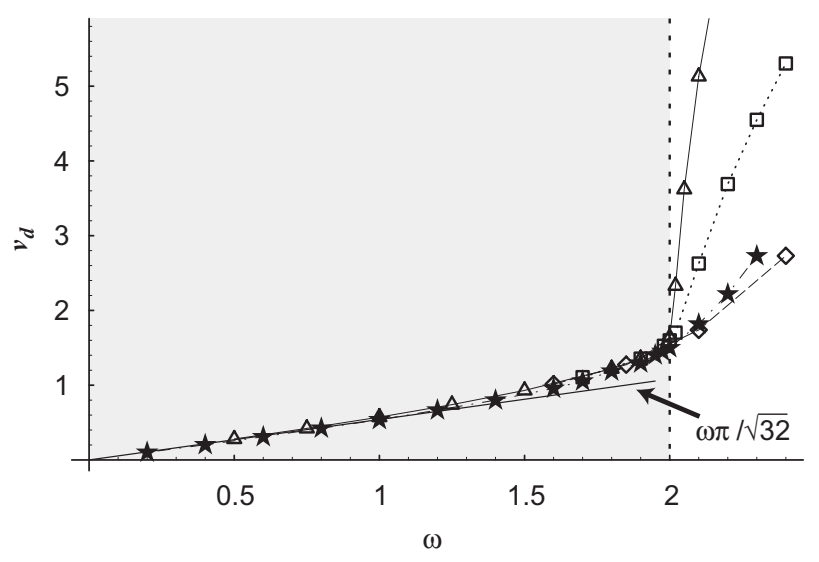

Fig. 13. The velocity of kinks vs. $\omega$ for a state with two kinks and different system size $L: \diamond=25, \star=30, \square=50, \triangle=100$. We have simulated Eq. (23) with $\mu=10$ and $q=0.1$. The dashed vertical line indicates the loss of equilibria (or SNIPER) at $\omega=2$. For small $\omega$ a linear relation between $\omega$ and $v_{\mathrm{d}}$ can be calculated. This result, Eq. (37), is shown by the tilted line.

We also note that up to $\omega=2$ the curves for different system size $L$ are very close to each other. This changes remarkably for values of $\omega$ beyond 2 where the slope of the curves depends strongly on $L$. In the limit $L \rightarrow \infty$ the curves approach a vertical line at $\omega=2$. One further finds that the kink velocity in the regime $|\omega|>2$ grows linearly 


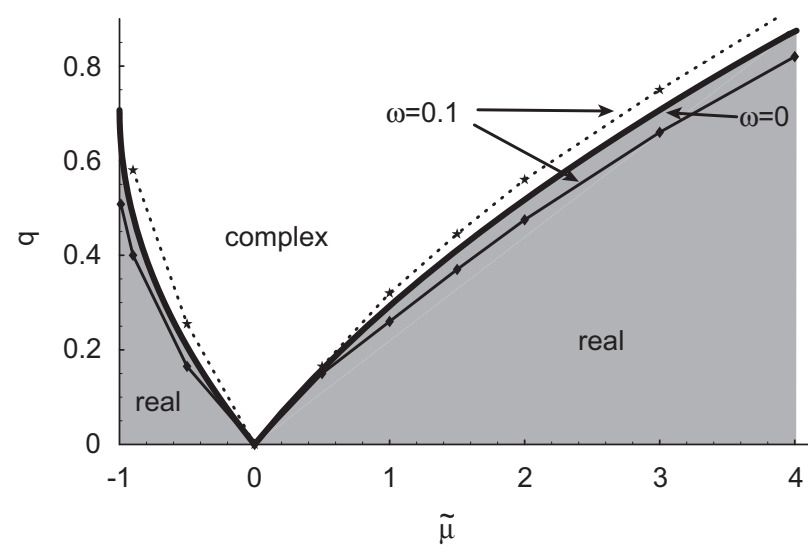

Fig. 14. For $\omega=0$ all eigenvalues are real in the gray area below the bold line. For $\omega=0.1$ the thin solid and the dotted lines represent the locations where a pair of eigenvalues becomes complex (numerical convergence was not sufficient for the dotted line close to $\tilde{\mu}=-1$ ). By numerical simulation of the full amplitude equation we found that a transition to oscillating kink interaction occurs at the dotted line.

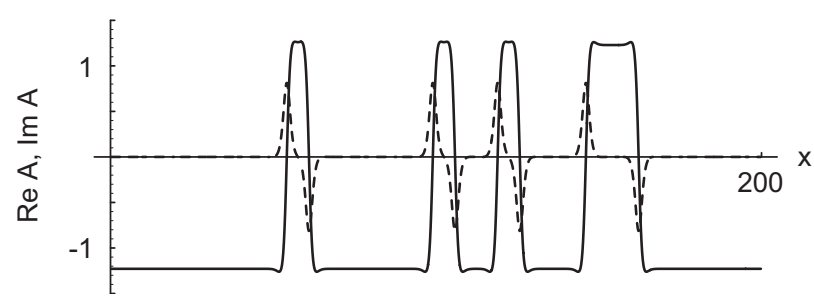

Fig. 15. A solution with several defects placed at different stable distances from each other $(\mu=1, q=0.7, \omega=0$, solid—Re $A$, dashed-Im $A)$.

with the distance between kinks if this distance is not too small. An explanation of this behavior using the phase approximation is given in the next section. Furthermore, the solution becomes less localized and more harmonic in space if one increases $\omega$, which can be attributed to the absence of fixed points of the homogeneous problem for $|\omega|>2$.

Interaction of kinks was studied in $[28,47]$. In a linearization of the ODEs $(27-30)$ four eigenvalues determine the asymptotic decay of fixed points and consequently the kink interaction for sufficiently distant kinks. If $\omega=0$, two pairs of eigenvalues with opposite sign exist $\left(\lambda_{1}, \lambda_{2},-\lambda_{1},-\lambda_{2}\right)$. Similar to the case $n=1$, one finds a transition from interaction, which is monotonic with distance, to an interaction, which oscillates with distance. In other words, beyond this transition the eigenvalues can be given by $\lambda_{1}, \lambda_{1}^{*},-\lambda_{1}$, and $-\lambda_{1}^{*}$, where $\lambda_{1}$ is complex. The transition is depicted by the bold line in Fig. 14, where the region of complex eigenvalues is white. Ideas on spatial chaos and its relation to dynamical systems theory therefore suggest that in this region one can find spatially chaotic arrays of kinks. An example for an aperiodic array of kinks, which is stable in numerical simulations of Eq. (23), is depicted in Fig. 15.

In [47] the consequences of a drifting forcing on kink interaction were studied. For non-zero $\omega$ the two pairs of eigenvalues become distinct not only by their sign but also by their absolute values. One now finds two curves where a pair of eigenvalues becomes complex. These curves are shown by the thin solid and dashed lines in Fig. 14. By numerical simulation of the full amplitude equation we found that a transition to oscillating kink interaction (indicated by the existence of bound pairs of kinks) occurs at the dotted line. We thus conclude that the range of complex kink interaction is reduced for increasing $\omega$. Following the ideas on spatial chaos and taking into account that for $\omega \neq 0$ the kinks travel, we suggest that this system provides a mechanism for chaotic trains of kinks [47,71].

\subsection{Phase approximation}

Under certain conditions one can find a simplified description of the amplitude equation using the so-called phase approximation. For strong linear damping of amplitude modes one can assume that the amplitude adiabatically follows 


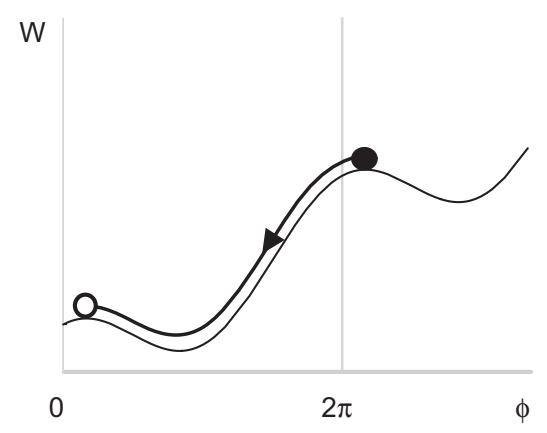

Fig. 16. Schematic plot of the potential $W$ in Eq. (36) and a heteroclinic connection between local extrema. The heteroclinic connection exists for a specific damping $v_{\mathrm{h}}$ only. If $v_{\mathrm{d}}$ is larger than $v_{\mathrm{h}}$ the trajectory will approach a local minimum. If $v$ is smaller than $v_{\mathrm{h}}$ the trajectory will be periodic.

the dynamics of the phase $\Theta$ (see Eqs. (24), (25)). This has been studied in the current context in [44]. For a recent review of the related case of forcing in oscillatory systems see Ref. [4].

The phase approximation is valid for sufficiently large $\mu$ (or, equivalently, for small forcing strength). Then the variation of $R$ is small and the fourth and fifth term on the rhs of Eq. (25) can be neglected:

$$
\frac{\partial \Theta}{\partial t}=\frac{\omega}{n}-R^{n-2} \sin n \Theta+\frac{\partial^{2} \Theta}{\partial x^{2}} .
$$

For instance, in the case of $n=2$ and $\omega=q=0$, one can see by comparing the $X$ and $Y$ components in Eq. (33) that $R$ is indeed approximately constant if $\mu$ is sufficiently large.

To simplify the analysis of Eq. (34) we rewrite it using $\phi=n \Theta$ :

$$
\frac{\partial \phi}{\partial t}=\omega-M \sin \phi+\frac{\partial^{2} \phi}{\partial x^{2}} .
$$

We treat $M \equiv n R^{n-2}$ as constant and independent of $x$. To determine kink solutions of the phase equation (35), we again address uniformly traveling solutions $\phi(x, t)=\phi(y \equiv x-v t)$. We find from Eq. (35):

$$
0=\frac{\partial^{2} \phi}{\partial y^{2}}+v \frac{\partial \phi}{\partial y}+\frac{\partial W}{\partial \phi}
$$

where we have introduced the potential $W \equiv M \cos \phi+\omega \phi$. Eq. (36) describes the evolution of the angle for a damped pendulum subject to a constant torque where $y$ plays the role of time. A brief discussion of its solution behavior is in order.

Assuming first that $|\omega|<|M|$ local extrema of the potential $W$ exist. Depending on $v$ there are three different types of behavior: if $v>v_{\mathrm{h}}$, the "friction" term $v \partial \phi / \partial y$ is strong enough so that the system will be trapped in the next local minimum. At a critical $v=v_{\mathrm{h}}(\omega)$ a connection between two local maxima exists (heteroclinic connection, see Fig. 16). If $v$ is decreased below $v_{\mathrm{h}}$ there exists an attracting periodic solution (where the variable $\phi$ is taken to be $2 \pi$-periodic, [67]).

Relating now the solutions of the amplitude equation with the solutions of the damped pendulum equation, we can identify the heteroclinic connection of the pendulum equation with a traveling solitary kink and the periodic solutions with traveling multi-kink states. Furthermore, in the amplitude equation the damping $v$ corresponds to the velocity $v_{\mathrm{d}}$ of kinks. An isolated kink should therefore exhibit a velocity $v_{\mathrm{h}}$. One also expects that the velocity $v_{\mathrm{d}}$ will be close to $v_{\mathrm{h}}$ for multi-kink states with sufficiently large distance between the kinks.

The critical $v_{\mathrm{h}}(\omega)$ that yields the heteroclinic connection in Eq. (36) depends in a non-trivial way on the slope $\omega$ of the potential. Except for the limit of small $\omega$ the heteroclinic orbit has to be determined numerically [72]. For $\omega \ll 1$ one can approximate the bifurcation curve of the heteroclinic connection linearly by [67]

$$
v_{\mathrm{h}}(\omega)=\omega \pi / \sqrt{16 M}
$$


We will now compare with simulations of the amplitude equation for 2:1 resonance. The velocity $v_{\mathrm{d}}$ of kinks for different choices of the domain length was shown in Fig. 13. Indeed for $\omega<2$ the velocities do not depend strongly on $L$ and provide a good approximation of $v_{\mathrm{h}}$. In this $\omega$-range the graph very much resembles the numerical findings for the critical damping rate of the heteroclinic connection in the damped pendulum equation (36) (see e.g. Fig. 3 in [73]). As discussed above, the linear relation between $\omega$ and $v_{\mathrm{h}}$ can be calculated perturbatively for small $\omega$ and reads $v_{\mathrm{h}}=\omega \pi / \sqrt{32}$. This tangent has been added in Fig. 13 .

Next we discuss the case $|\omega|>M$, where heteroclinic connections together with the fixed points are lost. The periodic solution (that is, the periodic array of kinks) that exists for $v<v_{\mathrm{h}}$ continues to exist if $|\omega|$ is increased beyond $M$ where the local extrema disappear (loss of fixed points or-in the amplitude equation-SNIPER). In this case the pendulum will approach a limit cycle. In the original system, the period of the pendulum corresponds to the distance between the kink-like local perturbations. In the limit of large $|\omega|$ (or large torque on the pendulum) the damping has to be strong enough to balance the potential force and an approximation of the distance between the kink-like perturbations of the pattern becomes possible by neglecting the inertial term in (36):

$$
v \frac{\partial \phi}{\partial y}-M \sin \phi+\omega=0 .
$$

Then the period of the periodic solution, and thus the distance between the kinks, can be determined analytically [67]:

$$
d=\frac{2 \pi v}{\sqrt{\omega^{2}-M^{2}}} .
$$

Comparison with numerical results for the amplitude equation such as those in Fig. 13 shows good agreement with approximation (39) for large $L=2 d$. For even larger values of $\omega$ the argument leading to Eq. (38) can be extended. In this case the "ripples" of the potential in the phase equation become negligible and we obtain $v \partial \phi / \partial x+\omega=0$. We can approximate $\partial \phi / \partial x \approx 1 / L$ and get from the phase equation

$$
|v| \approx \omega L .
$$

We thus find that the curves in Fig. 13 become linear again for very large $\omega$.

The phase approximation at sufficiently large $\mu$ can also be used for general $n$. The case $n=1$ has been considered in [44]. Similar to the case $n=2$, the pulses studied in Section 4.1.2 and their drift velocity can be characterized by the phase equation. In contrast to the $n=2$ case the critical $\omega$ for the loss of fixed points now depends on $\mu$.

\section{Two-dimensional patterns. Stripes and hexagons}

Let us now address the effects of the traveling wave forcing in two-dimensional patterns. We will first briefly consider possible modulational instabilities of entrained stripes. Afterward, we will analyze in detail instabilities leading to hexagonal patterns in 1:1 resonance.

\subsection{Modulational instabilities of entrained stripes}

The only inhomogeneous two-dimensional patterns that we will discuss are those caused by modulational instabilities of entrained stripes, as governed by Eq. (7). Let us consider a small periodic perturbation of the homogeneous striped pattern of amplitude $A_{0}=S \mathrm{e}^{\mathrm{i} \psi}$ entrained to the forcing with a mismatch $q$. Contrary to the usual Eckhaus instability analysis, here the deviation from the intrinsic wave-number $k_{\mathrm{c}}$ is fixed. After linearization about this entrained solution, the small, periodic perturbation of wave-number $\left(k_{x}, k_{y}\right)$ yields a growth rate of the form

$$
\lambda_{ \pm}=\mu-2\left|A_{0}\right|^{2}-\left(q+k_{y}^{2}\right)^{2}-k_{x}^{2} \pm \sqrt{\left|\epsilon(n-1)\left(A_{0}^{*}\right)^{n-2}-A_{0}^{2}\right|^{2}+\left(\frac{\mathrm{i} \omega}{n}-2\left(q+k_{y}^{2}\right) k_{x}\right)^{2}} .
$$

For $\omega=0$ this corresponds to the case of purely spatial forcing, which has been discussed in Ref. [27]. Note that in the long wavelength limit $\left(k_{x}=k_{y}=0\right)$ we recover the stability analysis of the fixed points (homogeneous patterns). We focus on the case of stable entrained stripes so the growth rate of the zero-mode is assumed negative. 
For $\omega \neq 0$ pure zig-zag modes $\left(k_{x}=0\right)$ may pick up an imaginary part of $\lambda$ at finite $\omega$. For $n=1$ the condition for that is $|\omega|>S^{2}$. Consequently, for small $|\omega|$ they do not propagate unless they are mixed with Eckhaus modes $\left(k_{x} \neq 0\right)$, but in general we may expect zig-zag waves. For the case $n=1$, the explicit dispersion relation for pure zig-zag modes reads

$$
\lambda_{+}=\mu-2 S^{2}+\left(S^{4}-\omega^{2}\right)^{1 / 2}-\left(q+k_{y}^{2}\right)^{2} .
$$

Contrary to the usual case, in the long-wavelength limit ( $k_{y}$ small) the zig-zag modes are stable. However, for $q<0$ there is a maximum of $\lambda$ at $k_{y, \max }^{2}=|q|$, so for a finite range of $k_{y}$ and sufficiently large $|q|$, zig-zag modes may become unstable. The condition $\operatorname{Re} \lambda_{+}\left(k_{y, \max }\right)=0$ then reads

$$
\mu-2 S^{2}+\operatorname{Re}\left[\left(S^{4}-\omega^{2}\right)^{1 / 2}\right]=0 .
$$

Therefore, depending on the parameters, the entrained striped solution may support either stationary zig-zag modes or zig-zag waves propagating in the transversal direction. Such transversal zig-zag waves have indeed been observed in experiments for large $\omega$ in the CDIMA reaction (see Section 6.4). Note that at exact resonance, $q=0$, zig-zag modes are always stable, so the existence of sustained (not damped) transversal waves in the experiment is a signature of the existence of a wave-number mismatch, a parameter which may not be easy to know experimentally.

On the other hand, Eckhaus modes $\left(k_{y}=0\right)$ always pick up an imaginary, propagative component for $\omega \neq 0$. In order to check their stability, let us consider first the case $\omega=0$. Then we obtain

$$
\lambda_{ \pm}\left(k_{x}\right)=\mu-q^{2}-2 S^{2}-k_{x}^{2} \pm \sqrt{4 q^{2} k_{x}^{2}+\left(\epsilon(n-1) S^{n-2}-S^{2}\right)^{2}} .
$$

Here we have used that stable solutions for $\omega=0$ are given by $A_{0}=S \exp (\mathrm{i} 2 \pi p / n)$, where $p$ is an integer number. A maximum growth rate $\lambda_{+}$is obtained for

$$
k_{x, \max }^{2}=q^{2}-\left(\epsilon(n-1) S^{n-2}-S^{2}\right)^{2} / 4 q^{2} .
$$

Then the condition $\lambda_{+}\left(k_{x, \max }\right)=0$ determines a transition surface

$$
\mu-2 S^{2}+\left(\epsilon(n-1) S^{n-2}-S^{2}\right)^{2} / 4 q^{2}=0 .
$$

For $n=1$ and $\epsilon=1$ this transition is shown in Fig. 8. For non-zero $\omega$ and in the long-wavelength limit we have

$$
\lambda=\mu-2 S^{2}+\left(S^{4}-\omega^{2}\right)^{1 / 2}-\mathrm{i} \omega \frac{2}{S^{2}} q k_{x}+\left(-1+\frac{2 q^{2}}{S^{2}}-\frac{2 \omega^{2} q^{2}}{S^{6}}\right) k_{x}^{2}+\cdots
$$

Again we see that, in contrast to zig-zag modes, Eckhaus eigenvalues possess an imaginary part, even for small $\omega$. Eckhaus modes for finite $k_{x}$ may therefore result in the existence of longitudinal waves. These have been observed in the experiments described in Section 6.4.

\subsection{Transitions between stripes and hexagons at 1:1 resonance}

For the rest of Section 5 we turn to the set of homogeneous amplitude equations (8-10) for 1:1 resonance. They were discussed in Section 2.2 and formulated as an autonomous system, with spatially homogeneous amplitudes. There we saw that a reduction of these three equations is possible if the lateral modes have equal amplitude. Here we will analyze the set of coupled amplitude equations $(11,12)$.

Through this subsection, and without loss of generality, we will use a scaling for which $\mu=1$. Consequently, we will have two free parameters $\epsilon^{\prime}$ and $\omega^{\prime}$. This is obtained from Eqs. $(11,12)$ by rescaling $t, A, B$ and the model parameters with $h^{\prime} \equiv \bar{h}=h / g, \alpha^{\prime} \equiv \bar{\alpha} / \sqrt{|\mu|}=\alpha / \sqrt{|\mu| g}, \epsilon^{\prime} \equiv \bar{\epsilon} /|\mu|^{3 / 2}=\sqrt{g} \epsilon /|\mu|^{3 / 2}, t^{\prime} \equiv|\mu| t$ and $\omega^{\prime} \equiv \omega /|\mu|$ (note that we are assuming here that $\mu>0$ ). In this representation, the coupled equations take the form

$$
\begin{aligned}
& \partial_{t^{\prime}} A=\left(1+\mathrm{i} \omega^{\prime}-|A|^{2}-2 h^{\prime}|B|^{2}\right) A+\alpha^{\prime} B^{* 2}+\epsilon^{\prime}, \\
& \partial_{t^{\prime}} B=\left(1-\mathrm{i} \omega^{\prime} / 2-|B|^{2}-h^{\prime}\left(|A|^{2}+|B|^{2}\right)\right) B+\alpha^{\prime} B^{*} A^{*} .
\end{aligned}
$$




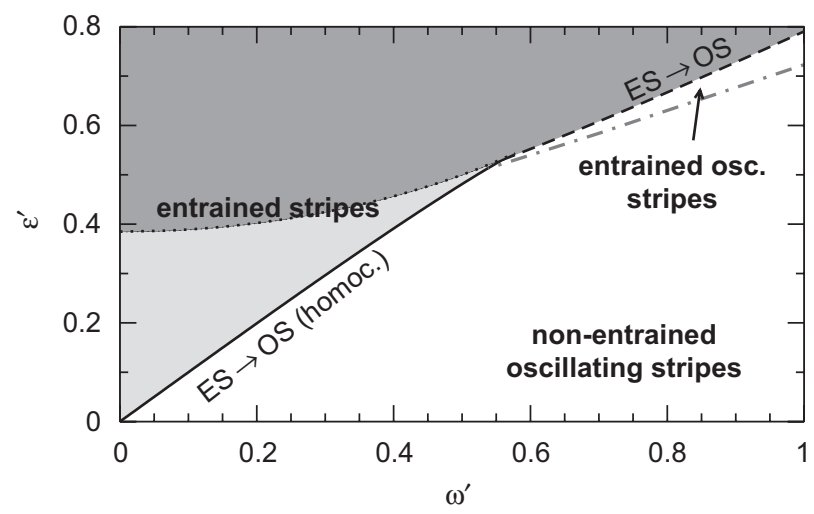

Fig. 17. Regions of stability in the parameter space $\left(\epsilon^{\prime}, \omega^{\prime}\right)$ where entrained stripes and oscillating stripes are stable (see also Fig. 3(a)), as calculated form the amplitude equation (48) with $B=0$. Entrained stripes are stable in the gray regions. The thick full- and dashed-lines indicate the loss of stability of the entrained stripes (SNIPER and Hopf bifurcations, respectively). The dot-dashed line separates the regions where the oscillating stripes are either entrained or non-entrained to the external forcing. The multistability region ends in a critical point located at $\omega^{\prime}=1 / \sqrt{3}$ and $\epsilon^{\prime}=\sqrt{8 / 27}$.

\subsubsection{Transition from entrained to oscillating stripes}

If the cross-coupling parameter $h^{\prime}$ in Eqs. $(48,49)$ is relatively large, then the mutual suppression between different modes is so strong that only stripes can be stable. Here we consider shortly this case and consequently assume $B=0$. Then, only the parameters $\epsilon^{\prime}$ and $\omega^{\prime}$ are relevant. This case corresponds to the homogeneous solutions of the zerodimensional problem discussed in Section 3.2. Here we want to interpret the results of that section in the context of the actual patterns that one observes in an extended systems.

Let us recall that for small values of $\epsilon^{\prime}$ and $\omega^{\prime}$ there is a region where three real solutions of Eq. (20) exist as shown in Fig. 17. These three solutions (a stable fixed point, a saddle and a repellor) are created and destroyed by saddle-node bifurcations. Outside this region a unique fixed-point solution exists, which becomes Hopf unstable at the dashed line in Fig. 17. This bifurcation corresponds to a transition from entrained stripes to oscillating stripes (i.e. stripes with a time modulated amplitude) and is denoted by ES $\rightarrow$ OS. This instability has been observed experimentally and numerically in the context of the CDIMA reaction in [40] (see discussion in Sections 6.2 and 6.3.1 below). Close to the bifurcation, modulations of the pattern amplitude are small, but as $\epsilon^{\prime}$ is decreased the modulations grow in size and eventually cross the origin in the complex $A$-plane. Then the velocity of the pattern starts to deviate from that of the forcing, and the stripes are thus not entrained. This transition curve is indicated by a gray dot-dashed line in Fig. 17 and corresponds to a transition from entrained to non-entrained stripes discussed in Section 3.2.

A second bifurcation leading to (non-entrained) oscillating stripes was described in Section 3. This global bifurcation, called SNIPER, gives rise to the transition from a stable fixed point to a finite-amplitude limit cycle. This bifurcation is indicated with a full line in Fig. 17 and is denoted by ES $\rightarrow$ OS (homoc.). For more details about stripe bifurcation in $1: 1$ resonance, we remit to Section 3.2.

\subsubsection{Emergence of hexagons}

Let us now address instabilities of entrained stripes leading to hexagonal patterns. A simple analysis of the stability of solutions with $B=0$ in Eqs. $(48,49)$, shows that entrained stripes will be stable against perturbations with non-zero $B$ as long as the conditions

$$
\begin{aligned}
& h^{\prime} S^{2}-1>0, \\
& \left(h^{\prime} S^{2}-1\right)^{2}+\omega^{\prime 2} / 4-\alpha^{\prime 2} S^{2}>0
\end{aligned}
$$

are both satisfied simultaneously. The breakdown of one or both of these conditions gives rise to a pattern with $B \neq 0$, which signals the presence of hexagonal symmetry. Let us now investigate each condition separately.

Condition (50) is not fulfilled if $\epsilon^{\prime}$ gets smaller than $\epsilon_{\mathrm{OH}}^{\prime}=\sqrt{\left(\left(1 / h^{\prime}-1\right)^{2}+\omega^{\prime 2}\right) / h^{\prime}}$. This instability leads to a Hopf bifurcation of the entrained stripes with frequency $\Omega_{\mathrm{OH}}=\sqrt{\omega^{\prime 2} / 4-\alpha^{\prime 2} / 2}$ and has $B \neq 0$. A necessary condition for the 
a

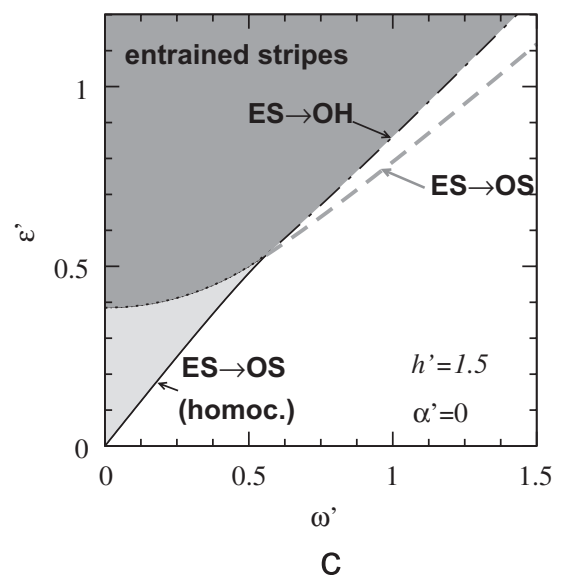

b

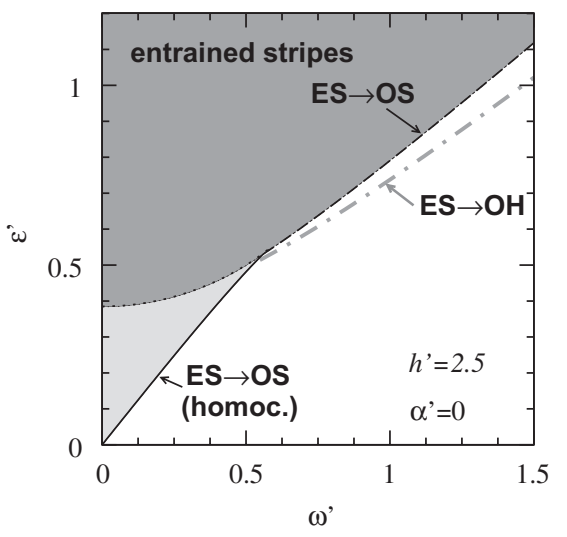

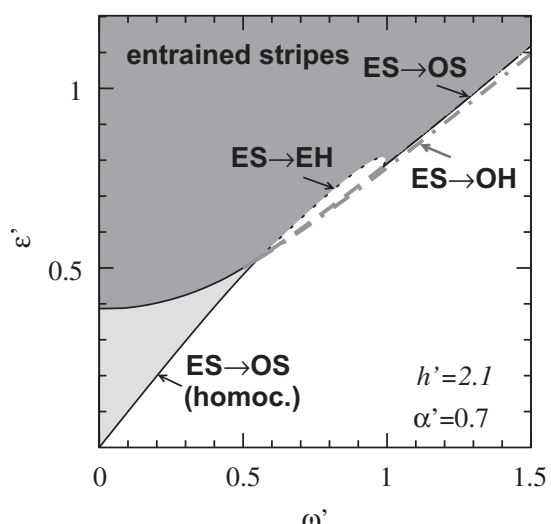

$\omega$

Fig. 18. Instabilities of entrained stripes in $2 \mathrm{~d}$. The gray areas correspond to regions where entrained stripes are stable. The dot-dashed line shows the location of the instability leading to oscillating hexagons occurring if $S^{2}<h^{\prime} / 2$ (cf. Eq. (50)). The dashed line shows the ES $\rightarrow$ OS bifurcation occurring if $S^{2}<1 / 2$. Note that in (a) for $1<h^{\prime}<2$ the $\mathrm{ES} \rightarrow \mathrm{OH}$ instability preempts the ES $\rightarrow$ OS bifurcation leading to oscillating stripes. The opposite is true if $h^{\prime}>2$, as exemplified in (b). In both cases $\alpha^{\prime}=0$. In (c) the ES $\rightarrow$ EH transition is also shown for $h^{\prime}=2.1$ and $\alpha^{\prime}=0.7$ with a thick dotted line. A more detailed analysis of this last case is shown in Fig. 21.

occurrence of this instability is that $\left|\omega^{\prime}\right|>\sqrt{2}\left|\alpha^{\prime}\right|$. It defines a transition from entrained stripes to oscillating hexagons and will be denoted as ES $\rightarrow$ OH in the following. This bifurcation is similar to the Hopf bifurcation ES $\rightarrow$ OS leading to oscillating stripes, but now with $B \neq 0$.

We will now discuss how the bifurcations ES $\rightarrow \mathrm{OH}$ and $\mathrm{ES} \rightarrow \mathrm{OS}$ are related to each other. Depending on the value of the coefficient $h^{\prime}$ (which describes the strength of the cross-interaction between the $A$ - and $B$-modes), either the ES $\rightarrow$ OH bifurcation or the standard Hopf ES $\rightarrow$ OS can occur first. More specifically, if $1<h^{\prime}<2$, then the bifurcation ES $\rightarrow \mathrm{OH}$ preempts the normal Hopf. The opposite is true if $h^{\prime}>2$. The special case $h^{\prime}=2$ corresponds to a degenerated situation. In Figs. 18(a) and (b), the line where the ES $\rightarrow$ OH bifurcation and the standard Hopf ES $\rightarrow$ OS occur are shown with dot-dashed and, respectively, dashed curves for two typical situations. Note also that the bifurcation threshold $\mathrm{ES} \rightarrow \mathrm{OH}$ is independent of the value of the parameter $\alpha^{\prime}$.

In Fig. 19 we show an example of the pattern arising after the breakdown of condition (50). Here we can see that the pattern oscillates between a hexagonal structure composed of white dots in a dark background and the opposite. Note also that the projections of the limit cycle in the $A$ - and $B$-planes have different frequency. Indeed, in the $B$-plane the frequency is $\Omega_{\mathrm{OH}}$ whereas in the $A$-plane it is $2 \Omega_{\mathrm{OH}}$. Also a time shift of half a period transforms $(A, B) \rightarrow(A,-B)$.

The breakdown of condition (51) is more difficult to analyze. This bifurcation is stationary (pitchfork) and it leads to entrained hexagons (ES $\rightarrow \mathrm{EH}$ in the following). These entrained hexagons occur in two symmetry degenerated variants related by $B \rightarrow-B$. This symmetry corresponds to a shift of the pattern by $1 / \sqrt{3}$ along the $y$-direction. 


\section{ARTICLE IN PRESS}

a

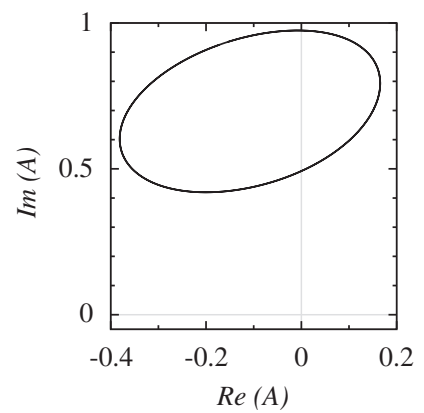

d

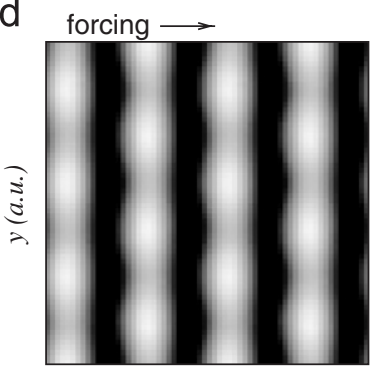

$x($ a.u. $)$ b

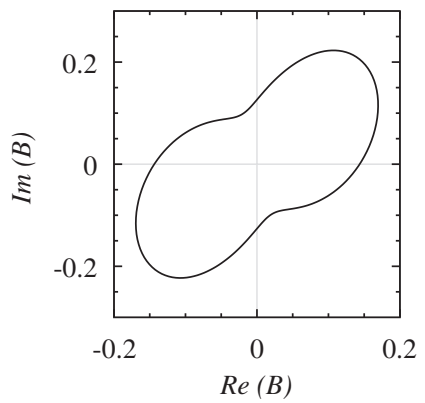

e

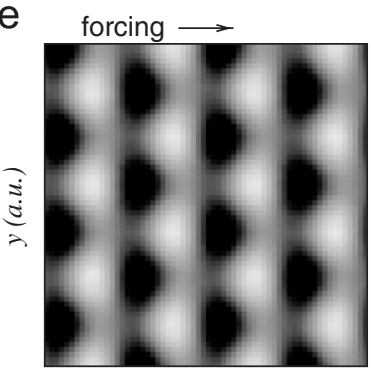

$x($ a.u.)
C

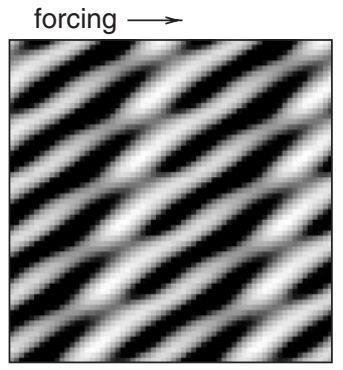

$x($ a.u. $)$

$f$

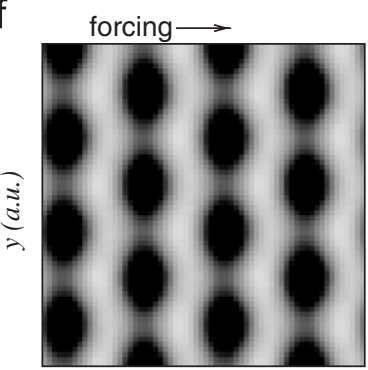

$x($ a.u.)

Fig. 19. Example of oscillating hexagons arising from the $\mathrm{ES} \rightarrow \mathrm{OH}$ bifurcation (with $h^{\prime}=1.8, \alpha^{\prime}=0.7, \epsilon^{\prime}=0.9$ and $\omega^{\prime}=1.2$ ). In (a) and (b) the projections of the limit cycle in the planes $A$ and $B$ of Eqs. $(48,49)$ are shown. The plots (d), (e) and (f) show three consecutive snapshots of the corresponding patterns. A space-time plot along a horizontal cut of these snapshots is shown in (d).

a

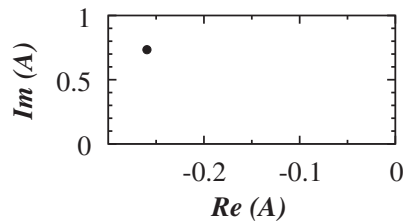

C

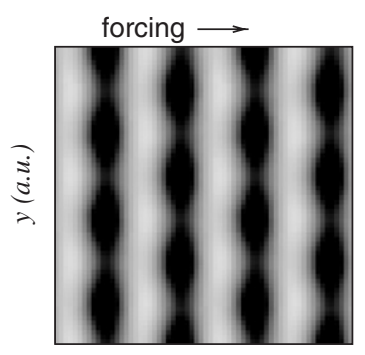

d

b

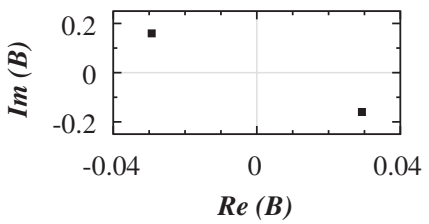

$x($ a.u. $)$

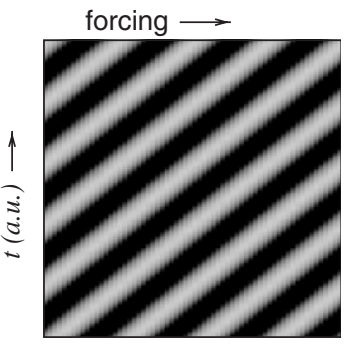

$x($ a.u. $)$

Fig. 20. Example of entrained hexagons arising from the ES $\rightarrow$ EH bifurcation (with $h^{\prime}=2.1, \alpha^{\prime}=0.7, \epsilon^{\prime}=0.7$ and $\omega^{\prime}=0.83$ ). The location of the corresponding fixed points of Eqs. $(48,49)$ in the planes $A$ and $B$ is shown in (a) and (b). In plot (c) we show a snapshot of the reconstruction of the original pattern after the ES $\rightarrow$ EH bifurcation. In (d) a spatio-temporal plot corresponding to a horizontal cut of (c) is shown.

Violation of condition (51) can only occur inside an interval of $\omega^{\prime}$. Indeed, $\omega^{\prime}$ should be smaller than a certain threshold value. If $h^{\prime}<2$ then this maximal value is given by $\omega^{\prime 2}<\left(4 / h^{\prime}\right) \alpha^{\prime 2}$. Otherwise, for $h^{\prime}>2, \omega^{\prime 2}<\left(4 / h^{\prime}\right) \alpha^{\prime 2}$ should hold for the instability ES $\rightarrow$ EH to be possible. For the ES $\rightarrow$ EH instability interval to be non-zero, $\alpha^{\prime}$ must be larger than a certain minimal value, which depends on $h^{\prime}$. In Fig. 18(c), the ES $\rightarrow$ EH bifurcation takes place at the dotted line. Note that this bifurcation may preempt either the $\mathrm{ES} \rightarrow \mathrm{OS}$ or the $\mathrm{ES} \rightarrow \mathrm{OH}$ instabilities for intermediate values of $\omega^{\prime}$. In Fig. 20 a typical example of the pattern arising after the ES $\rightarrow$ EH bifurcation is shown. 


\subsubsection{Heuristic discussion of hexagons induced by stripe forcing}

Even if Eqs. (50) and (51) need to be solved to unfold the complete bifurcation scenario, one can gain some physical insight into the mechanism responsible for the emergence of hexagons with a simple heuristic argument.

We begin by discussing some properties of entrained stripe solutions given by Eq. (20). In fact, this fixed point equation defines a function $S\left(\omega^{\prime}, \epsilon^{\prime}\right)$ for each of the fixed points. It is important to realize that both parameters have typically competing effects, as can be argued on physical grounds, since increasing the velocity of the forcing should eventually average out the effect of the forcing itself. This will translate in the different dependence of the size of the response $S$ to both parameters.

In general, it can be proven that, whenever there is a single solution for $S^{2}$ in Eq. (20) (i.e. the dark gray area of Fig. 17), then $S\left(\epsilon^{\prime}, 0\right)>S\left(\epsilon^{\prime}, \omega^{\prime}\right)$. On the other hand, if there are three solutions of $S^{2}$, then both extremal solutions (i.e. the stable fixed point and the repellor) also satisfy $S\left(\epsilon^{\prime}, 0\right)>S\left(\epsilon^{\prime}, \omega^{\prime}\right)$. The opposite is true for the saddle fixed point which lies in between. This statement can be easily checked graphically by plotting the function $S^{2}\left(\epsilon^{2}\right)$ as defined by Eq. (20) for both $\omega^{\prime}=0$ and $\omega^{\prime} \neq 0$. Since the solution observed in a real system will be the stable one, the condition that $S\left(\epsilon^{\prime}, 0\right)>S\left(\epsilon^{\prime}, \omega^{\prime}\right)$ is satisfied in particular in that case. This means that, for a fixed $\epsilon^{\prime}$, the amplitude of the pattern will decrease with $\omega^{\prime}$.

This property of the stable solution is at the root of the emergence of the hexagonal symmetry. In fact, one expects that for increasing strength of the forcing $\epsilon^{\prime}$ at 1:1 resonance, the magnitude of the resonant mode will also increase. Since we assume to be in a situation where hexagons are not present, the three-mode $120^{\circ}$ resonant interaction weighted by $\alpha^{\prime}$ (responsible of the occurrence of hexagonal patterns) is depressed, that is, it is overcome by the cross-coupling interaction, weighted by $h^{\prime}$. As long as the forward mode $A$ is enhanced by a static forcing, the 3-mode interaction responsible for the hexagons would thus be further depressed. However, as one introduces the motion of the forcing, we have seen that the modulus of the 1:1 resonant mode $A$ with the forcing decreases for increasing $\omega$. This diminishes the effect of the term $-h^{\prime}|A|^{2} B$ which competes with the 3-mode resonance, thus favoring the emergence of hexagons. This may happen unless the quadratic terms in the amplitude equations which mediate the resonant three-mode interaction are absent (i.e. $\alpha=0) .{ }^{1}$ In this way, the above argument pictures the occurrence of hexagons roughly in terms of a shift of the pre-existing transition boundary between stripes and hexagons. However, notice that the presence of the imaginary parts in the linear terms for $\omega^{\prime} \neq 0$ introduces non-gradient dynamics into the system, resulting in new solutions which have no counterpart in the unforced system, such as the simplest oscillating hexagons resulting from a primary Hopf bifurcation. In the next section we will see that the time-dependent solutions that appear beyond the primary instabilities may exhibit rather complex behavior, including temporal chaos.

\subsubsection{Beyond primary instabilities}

In the previous subsections we have shown that three different patterns may arise as the entrained stripes become unstable: oscillating stripes (ES $\rightarrow \mathrm{OS}$ ), oscillating hexagons $(\mathrm{ES} \rightarrow \mathrm{OH})$ and entrained hexagons $(\mathrm{ES} \rightarrow \mathrm{EH})$. We now go beyond the thresholds of these primary instabilities. Consequently, we resort to a full numerical analysis of Eqs. $(48,49)$. We will show that one may expect different kinds of hexagonal patterns in addition to the ones reported so far.

Here we will restrict to the case $h^{\prime}>2$ with $\alpha \neq 0$, which is the one relevant for the existing experiments on the CDIMA reaction $[42,43,45]$. These experiments will be reviewed in detail in Section 6.4, here we just mention that it was experimentally observed that an increase in $\omega^{\prime}$ leads to an instability of the entrained stripes to oscillating stripes. This indicates that $h^{\prime}>2$ for those particular experiments. Note that the case $h^{\prime}<2$ could be of relevance for other experimental systems, but has not been addressed so far.

In this subsection we will consider in detail the case $h^{\prime}=2.1$ and $\alpha^{\prime}=0.7$. In Fig. 18(c) we have already shown the location of the primary instabilities in the parameter space. We saw that for $\omega^{\prime}$ high enough a ES $\rightarrow$ OS instability is expected as $\epsilon^{\prime}$ is decreased. The same applies for small values of $\omega^{\prime}$ (a ES $\rightarrow$ OS (SNIPER) bifurcation). For intermediate values the $\mathrm{ES} \rightarrow \mathrm{EH}$ instability occurs.

In Fig. 21 we summarize the outcome of extensive numerical simulations of Eqs. $(48,49)$ and in Fig. 22 some typical orbits are plotted. In the gray areas the entrained stripes are stable, whereas in the white region only oscillating stripes

\footnotetext{
${ }^{1}$ This would be the case in Boussinesq Rayleigh-Bénard convection. In that case, a different set of amplitude equations should be derived, suitable for the study of other states expected to arise, such as rectangular patterns. 
a

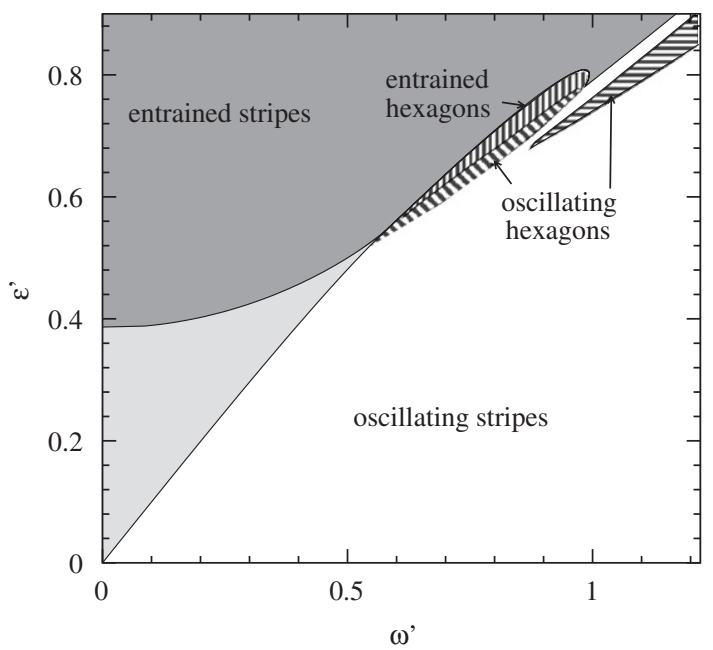

b

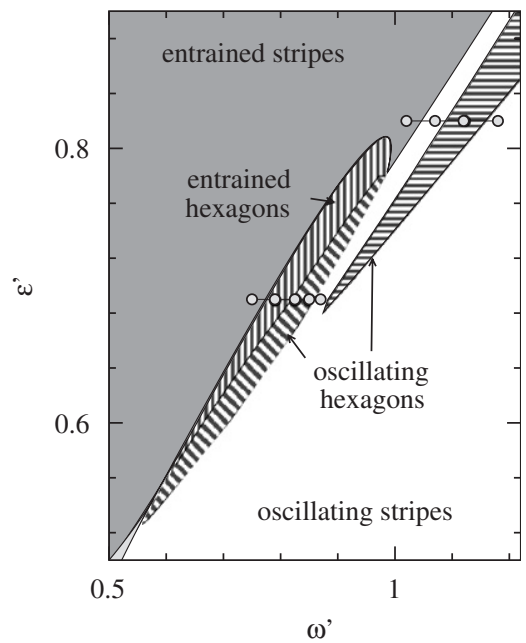

Fig. 21. Parameter space $\epsilon^{\prime}$ vs. $\omega^{\prime}$ showing the primary instabilities ES $\rightarrow$ OS and ES $\rightarrow$ EH for $h^{\prime}=2.1$ and $\alpha^{\prime}=0.7$ and the patterns arising after these instabilities. In hatched regions different kinds of hexagonal patterns are observed. In the vertically hatched region entrained hexagons are stable. They arise through a supercritical bifurcation of type ES $\rightarrow$ EH. For smaller values of $\epsilon^{\prime}$ we observe numerically a transition between the entrained hexagons and oscillating hexagons. These are stable in the slant-hatched region. A second unexpected region of oscillating hexagons is also observed in the horizontally hatched region. In (b) an enlargement of the region where hexagons are observed is shown. The horizontally ordered gray dots show the location of the phase-space plots shown in Fig. 22.

are stable. In the hatched regions hexagonal patters are stable. Let us discuss each of these regions where hexagons are stable in some detail.

In the vertically hatched region entrained hexagons are observed. These hexagonal patterns were the only ones predicted by the stability analysis discussed in the previous subsection. They arise through the ES $\rightarrow$ EH instability.

Quite unexpected are the hexagonal patterns observed in the slant- and horizontally hatched regions. They correspond to oscillating hexagons. Recall that for the value of $h^{\prime}$ under consideration here, they cannot appear form a primary instability. However, they arise from secondary bifurcations of either the entrained hexagons or the oscillating stripes. Two examples of these non-trivial bifurcation sequence are shown in Figs. 22(a) and (b) for two different values of $\epsilon^{\prime}$ (corresponding to two horizontal cuts in the parameter space of Fig. 21). Both of these oscillating hexagons are entrained to the forcing since the orbits do not enclose the origin $A=0$, as it is apparent in Figs. 22(a) and (b).

The oscillating hexagons in the horizontally hatched region, appear as a secondary bifurcation of the oscillating stripes, as $\omega^{\prime}$ increases. Their amplitude in the $B$-plane grows to reach a maximum, but as $\omega^{\prime}$ increases further, their amplitude decreases again until they disappear to return to the oscillating stripes.

Note also that there exist two different families of oscillating hexagons in the slant-hatched region. Depending on the initial conditions, the system will select either one or the other (i.e. they exhibit bistability).

Furthermore, the oscillating hexagons in the slant-hatched region can have complex dynamics. As $\epsilon^{\prime}$ is decreased, we have observed that near the critical point a cascade of bifurcations leading to chaos occurs. As $\epsilon^{\prime}$ is decreased further an inverse cascade is observed before the transition to oscillating stripes takes place. We have not studied these chaotic regimes in detail, but we would like to call the attention to this complex behavior and in particular to its extension to inhomogeneous patterns as an interesting line of study.

\section{Physical examples and experimental evidence}

\subsection{The photosensitive CDIMA reaction}

The theory of traveling-wave forcing has been developed in recent years in parallel with experiments on the so-called chlorine dioxide-iodine-malonic acid (CDIMA) reaction [40,42,43,45,46]. This is a well known reaction-diffusion 
a
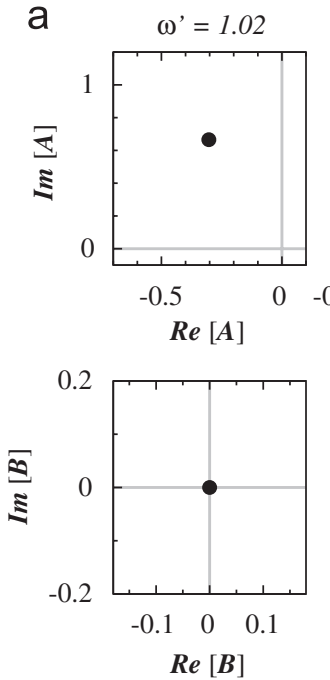

$\omega^{\prime}=1.07$
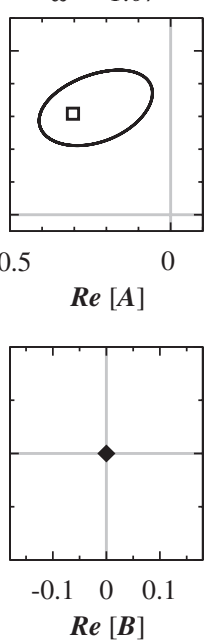

$\omega^{\prime}=1.12$

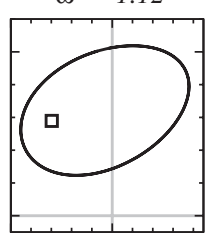

$-0.5$

$\operatorname{Re}[\boldsymbol{A}]$

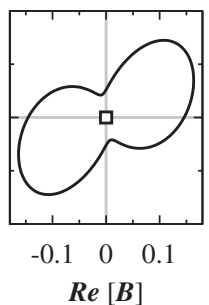

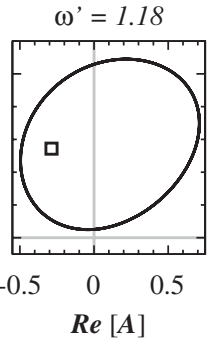

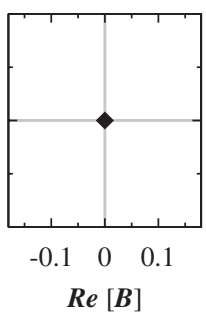

b

$\omega^{\prime}=0.75$
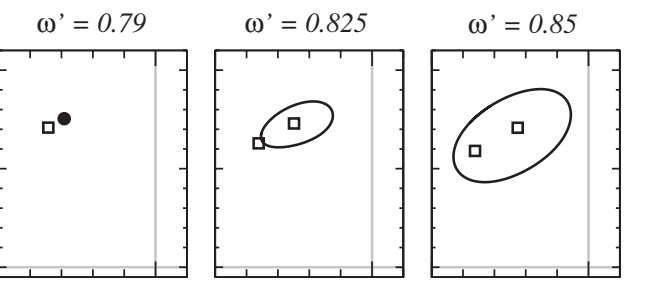

$\omega^{\prime}=0.87$
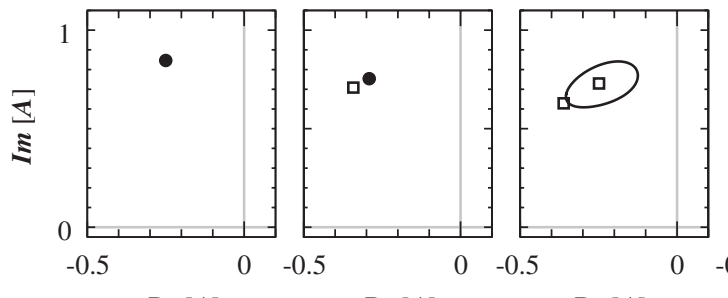

$-0.5$
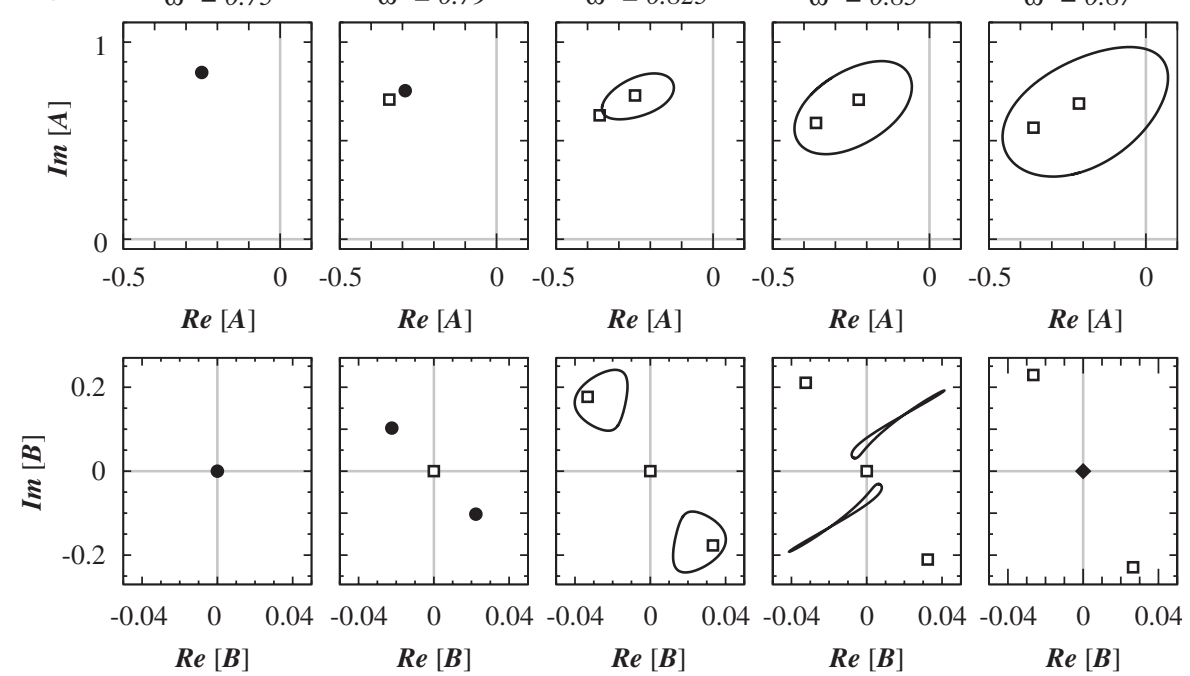

\section{$\operatorname{Re}[A]$}

$\operatorname{Re}[A]$

$\operatorname{Re}[A]$
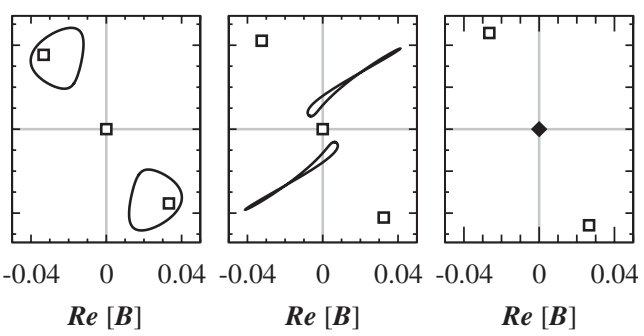

Fig. 22. Examples of the evolution of limit cycle orbits in Eqs. $(48,49)$ with $\epsilon^{\prime}=0.82$ in (a) and $\epsilon^{\prime}=0.69$ in (b) as $\omega^{\prime}$ is increased (with $\alpha^{\prime}=0.7$ and $h^{\prime}=2.1$ ). In Fig. 21(b) the location of these examples in the parameter space $\left(\omega^{\prime}, \epsilon\right)$ is displayed with open circles. The upper (lower) rows of (a) and (b) show the projection of the orbits in the $\operatorname{Re}[A]$ vs. $\operatorname{Im}[A]$ plane $(\operatorname{Re}[B]$ vs. $\operatorname{Im}[B]$ plane, respectively). Full circles, diamonds and thick black curves indicate the location of the stable fixed points and limit cycles in the $A$ - and $B$-planes (where the diamonds correspond to the projection of the stable limit cycles in the $B$-plane). The open squares show the projection of the unstable fixed points in $A$ - and $B$-planes. (Adapted form [43].)

system [58], which—in a quasi two-dimensional setup—-has become a prototype of the Turing instability [60-63], and which has the interesting property of being photosensitive [59]. In this way, it can be forced through illumination and is thus a very convenient model system to study spatio-temporal forcing. In fact, most of the analytical results on the amplitude equations for both $1 \mathrm{~d}$ and $2 \mathrm{~d}$ for the $1: 1$ resonance have been observed experimentally in the CDIMA reaction, or, in some cases, were preceded by experimental observations. Other predictions, such as those for 2:1 resonance, have not been explored yet. On the other hand, a varied range of interesting responses of this system has been obtained which are not understood and still call for new theoretical insights. A detailed, systematic study of different regimes has been recently reported in [45].

Depending on the values of control parameters, the CDIMA reaction exhibits either an oscillatory (Hopf) instability or a stationary (Turing) instability. Here we will only consider the regime, where patterns are generated by the original Turing mechanism [60-63], which results from the competition between autocatalytic reaction steps and the large 

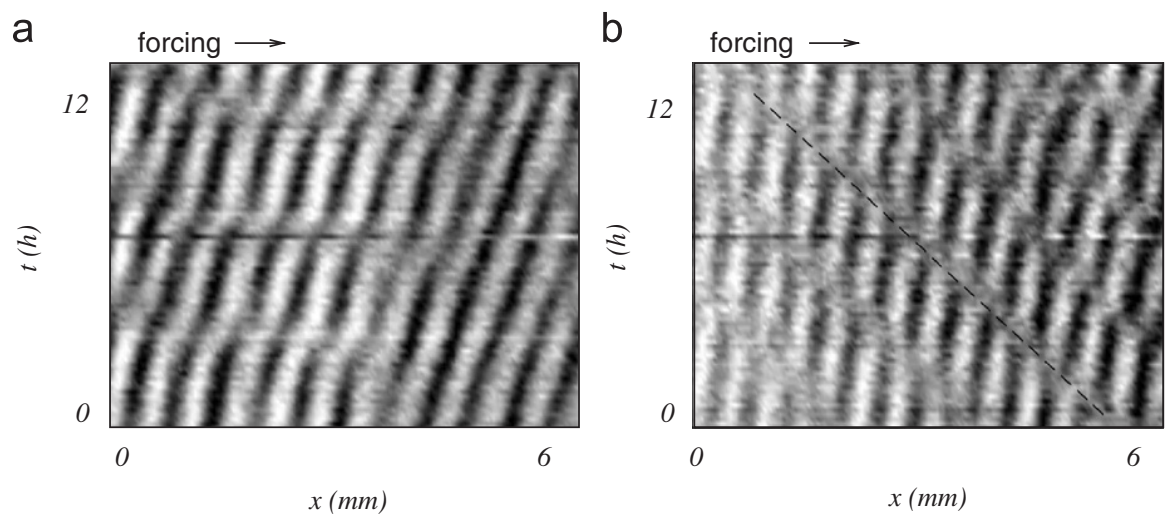

Fig. 23. Experimental space-time plots of an oscillating stripe solution (a) and a defect solution (b). The dashed line in (b) is a guide to the eye. The velocity of the forcing pattern is $v=0.13 \mathrm{~mm} / \mathrm{h}$ for both cases, the imposed wavelength in (b) is 1.1 times the spontaneous one. (Adapted from [40].)

contrast in diffusivity of two reactants. Even in the Turing regime, the reaction may exhibit hexagonal and striped patterns. Here we will always stay in the parameter regime where, with a uniform illumination condition, the only stable configuration is that of stripes. Then, the modulation of the light conditions will allow the local control of reaction rates at will, by focusing the desired spatio-temporal light pattern into the quasi two-dimensional reactor. A detailed description of the experimental setup for these studies can be found in Ref. [45].

The CDIMA reaction is usually modeled as a reduced set of reaction-diffusion equations within the Lengyel-Epstein (LE) scheme [58], modified to include the effect of illumination [59], as

$$
\begin{aligned}
& \partial_{t} u=a-c u-4 \frac{u v}{1+u^{2}}-\phi+\frac{\partial^{2} u}{\partial x^{2}}, \\
& \partial_{t} v=\sigma\left(c u-\frac{u v}{1+u^{2}}+\phi+d \frac{\partial^{2} v}{\partial x^{2}}\right) .
\end{aligned}
$$

Here $u$ and $v$ are the dimensionless concentrations of two of the chemical species that act as activator and inhibitor, respectively; $a, c, \sigma$, and $d$ denote dimensionless parameters of the chemical system. The effect of external illumination is introduced through the $\phi$-terms. This contribution is then decomposed into the mean value $\phi_{0}$ and a modulation part: $\phi(x, t)=\phi_{0}+\xi \cos \left(k_{f} x-\omega t\right)$. For purely homogeneous illumination, $\xi=0$, the equations admit a stationary solution, which in the following will be referred to as base state: $u_{0}=\left(a-5 \phi_{0}\right) /(5 c), v_{0}=a\left(1+u_{0}^{2}\right) /\left(5 u_{0}\right)$.

While the connection between experimental parameters and those appearing in the reaction-diffusion equations are well established empirically, the connection between the parameters in the reaction-diffusion model and those in the amplitude equation must be determined by standard methods of weakly nonlinear analysis. Those have only been obtained recently and are reported in Ref. [37].

\subsection{Experiments in quasi-one dimension near 1:1 resonance}

The first experimental evidence of entrained and oscillating patterns as well as defect states in the CDIMA reaction was reported in [40]. The controlled forcing was obtained through the projection of traveling light stripes. Under the chosen set of reagent concentrations, the system at dark spontaneously yields disordered stripe patterns with a wavelength of $0.54 \mathrm{~mm}$. In a typical experiment, parallel light stripes with a characteristic wavelength of precisely $0.54 \mathrm{~mm}$ were focused on the gel layer and were moved in the longitudinal direction with well-controlled and constant velocity $v_{f}$. For very small values, trivial traveling stripes, following adiabatically the imposed pattern, were found. As the passing velocity was increased they transformed into the modulated striped mode (see Fig. 23(a)). This transition can be interpreted in the context of the amplitude equation as the transition from entrained stripes to oscillating stripes. Furthermore, when considering a slight mismatch in the forcing wavelength, localized structures were observed, 
a

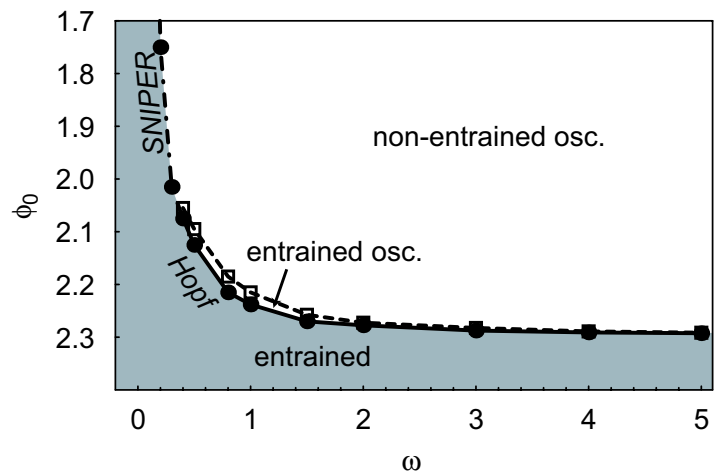

b

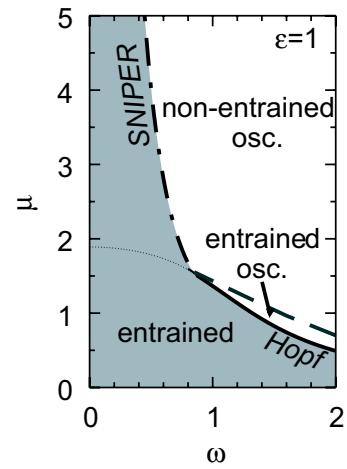

Fig. 24. Transition from entrained stripes to oscillating stripes in the LE model in (a) (with parameter values as described in the main text) and in (b), for comparison, in the amplitude equation (13) (with $\epsilon=1$ and $n=1$ ). In (a) the solid curve represents the transition to oscillatory states. It is mediated by a Hopf bifurcation for $\omega>0.3$ and the SNIPER bifurcation otherwise. Between the solid and the dashed curve one finds entrained oscillatory states. The dashed line shows the transition from entrained to non-entrained states.

propagating in the opposite direction to that of the stripes (see Fig. 23(b)). This result provided direct evidence for the existence of the predicted propagating localized perturbations in the chemical system.

The predictions reported in this review concerning the $2: 1$ case in $1 \mathrm{~d}$, have not been explored experimentally in any physical system yet.

\subsection{The Lengyel-Epstein model in one dimension}

We now demonstrate the existence of the dynamical regimes found for the amplitude equation for the set of reaction diffusion equations, which model the CDIMA reaction. All results were obtained through numerical integration of the LE equations $(52,53)$ with periodic boundary conditions by means of a pseudo-spectral method with a linearimplicit time-stepping [40]. From here on we fix the parameters to the following: $a=16, c=0.6, d=1.07$, and $\sigma=301$. These values were chosen to reproduce the experimental conditions for the CDIMA reaction. The large $\sigma$ value, corresponding to a strong diffusion contrast between the two species, guarantees the distance from the oscillatory regime of this chemical system. Consequently, only the Turing bifurcation will play a role.

The remaining parameters concern the forcing term. One of them, $\phi_{0}$, serves as the parameter to locate the position of the Turing bifurcation in the homogeneous problem. For the given parameters it occurs at $\phi_{0} \approx 2.3$ (the base state being unstable to Turing patterns below $\phi_{0} \approx 2.3$ ) and the critical wave-number is $k_{\mathrm{c}} \approx 1.07$.

\subsubsection{Exact 1:1 resonance}

We first discuss the case of exact 1:1 spatial resonance $k_{\mathrm{c}}=k_{f}$. Choosing the length $L$ of the periodic domain to be $10 \lambda$, where $\lambda=2 \pi / k_{\mathrm{c}}$ is the critical wavelength at instability, we fix $L=58.72$ and the forcing amplitude $\xi=0.1$.

As was discussed in the context of amplitude equations in Section 3, the 1:1 resonance yields an imperfect bifurcation to Turing patterns. Accordingly, the base state ceases to be a stationary solution and is modified into a non-trivial state for every value of the bifurcation parameter $\phi_{0}$. The first state we want to describe is a traveling, or entrained wave. The entrained wave locks to the forcing wave, adopting the same wave-number and frequency, with only a constant phase difference. This is the trivial state to be expected for slow driving and for large $\phi_{0}$. The entrained waves exist in the gray area of Fig. 24(a). The equivalent behavior in the amplitude Eq. (13) is shown in Fig. 24(b) for comparison.

Crossing the lower solid in Fig. 24(a) the entrained state undergoes a transition into a state with temporally modulated amplitude (oscillating stripes). Note that the modulation of the amplitude occurs uniformly in the entire system. The transition corresponds to a Hopf bifurcation for $\omega>0.3$ and the SNIPER bifurcation otherwise. For large $\omega$ the modulation frequency converges to the frequency $\omega$ of the forcing wave.

Furthermore, in accordance with results for the amplitude equation we find two types of oscillations. First, one finds solutions which are periodically modulated, but for which the average velocity of the pattern coincides with that of the 
a

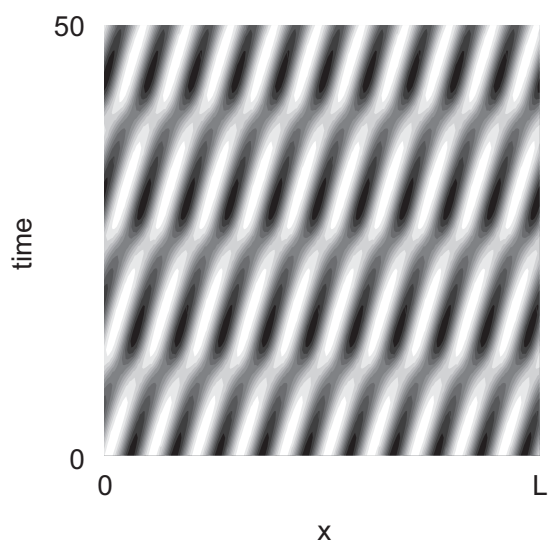

C

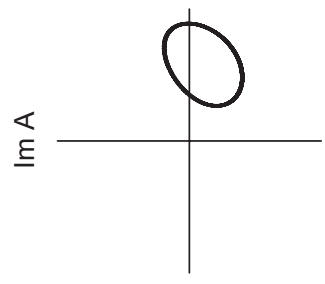

$\operatorname{Re} A$ b

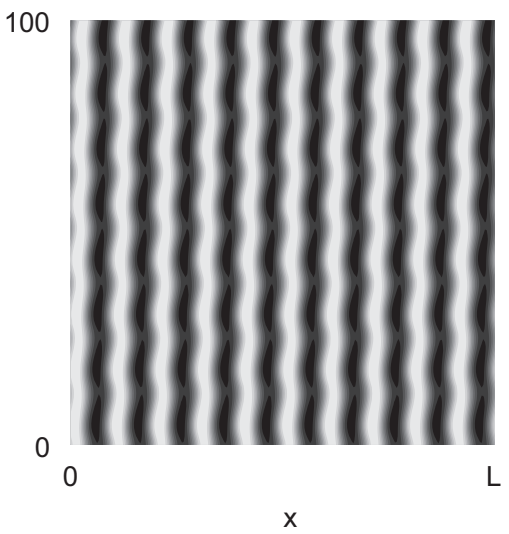

d

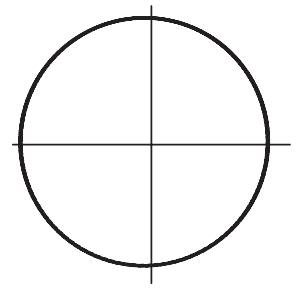

$\operatorname{Re} A$

Fig. 25. Space-time plots showing the $u$-component of oscillating states for $\omega=0.5, \phi_{0}=2.11$ (a) and $\phi_{0}=1.0$ (b). The length of the domain is $L=58.72$. The lower panels (c,d) show the corresponding limit cycle of the amplitude $A$ obtained by projection of the full solution onto the basic mode $A(t) \exp \left(\mathrm{i} k_{f} x-\mathrm{i} \omega t\right)$.

forcing (Fig. 25(a)). The existence range of these solutions is located in Fig. 24(a) between the solid and the dashed curve. Decreasing $\phi_{0}$ there is a further transition to states, which do not follow the forcing (Fig. 25(b)). Instead one finds an oscillation of the basic pattern and a drift, which is slower than the forcing's motion. These solutions exist above the solid and dashed curves in Fig. 24(a).

\subsubsection{Near 1:1 resonance}

Next we discuss defect states, or pulses, close to 1:1 resonance. In the Lengyel-Epstein model they have been reported in [40]. They manifest themselves for instance in space-time plots of the $u$-component as depression of the amplitude traveling on the background of a periodic pattern that moves with the forcing.

Rüdiger et al. [40] used simulations in the finite domain described in the previous section to determine the range of existence of stable non-homogeneous states. Localized states where found in two different regimes according to the forcing amplitude. In Section 4.1.2 it was shown that pulses only exist for sufficiently large $\mu$ (see Figs. 7 and 8). Because of the scaling used in that section, large $\mu$ corresponds to small forcing amplitude $\xi$ in the current context. Therefore, if $\xi$ is large, stable pulses do not exist. However, if the forcing frequency $\omega$ is too large for the stability of entrained states (above the instability to oscillating stripes, or SNIPER), non-homogeneous states exist, which resemble the proper pulses. An example of such a solution is shown in Fig. 26. On the other hand, for sufficiently small $\xi$, localized solutions in the LE model are stable even in the limit of vanishing $\omega$, i.e. in the stability domain of entrained stripes (see Fig. 4 in [40]). This regime clearly corresponds to the regime of pulses in the amplitude equation and therefore demonstrates the existence of pulse states in the LE model.

We finally mention further qualitative aspects of localized solutions in the LE model. It follows from the discussion of pulses within the amplitude equation, that localized perturbations can move with positive or negative velocity relative to the background pattern. States of propagation in both directions have also been observed in numerical simulations of the LE equations. Furthermore, the simulations showed that solutions with defects of different chiralities and different 


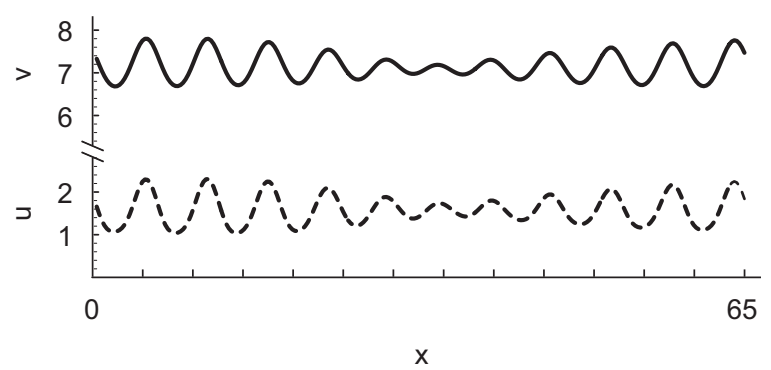

Fig. 26. A localized distortion for the LE model $\left(\omega=0.5, \phi_{0}=2.25, \xi=0.03, k_{f}=0.967\right)$. The underlying pattern moves to the right, while the distortion travels to the left.

spatial distances can coexist for a given set of parameters, which—from what was said in Section 4.1.2—is also possible in the amplitude equation.

In summary, these results show the applicability of our results for the amplitude equation, although a systematic analysis and comparison of defect states in the LE model remains to be done.

\subsection{Experimental results in two dimensions}

A detailed study of $2 \mathrm{~d}$ pattern dynamics in the CDIMA reaction under traveling-stripe forcing has been reported in Ref. [45], many of them still not understood. Here we will briefly review the experimental observations related to the phenomena predicted by the amplitude equations for hexagonal patterns, as described in Section 5.

At very small velocities of the forcing $v_{f}$ the problem is equivalent to the one-dimensional case discussed in Section 6.2. The patterns reported there were in fact two dimensional with no transversal variation. We thus observe the transition from entrained to oscillating stripes at exact 1:1 resonance.

Truly two-dimensional effects appear for moderate values of the forcing velocity $[42,43]$. The emergence of a hexagonal arrangement of the system under traveling-stripe forcing, either oscillating or entrained to the forcing was indeed first discovered experimentally in the CDIMA reaction. This occurred in a regime where this system does not support hexagons if it is unforced. In Fig. 27 we show results corresponding to high light intensities, resulting in the pattern referred in Section 5.2 as oscillating hexagons. The hexagonal lattice appears to oscillate at a frequency close to the one imposed by the forcing and alternates between patterns of white and black spots (Fig. 27(a) and, respectively, Fig. 27(c)).

For low light intensities a different kind of hexagonal patterns has been observed. In contrast to the previous case, this lattice is composed of black spots which move entrained with the forcing (see Fig. 28). We refer to this spatiotemporal structure as a pattern of entrained hexagons. Experiments show that this mode appears for a narrow range of parameters, while the oscillating hexagons mode seems to be more prominent.

Finally, if we further increase the velocity, the system adopts again a stripe-like mode, just as a precursor of the natural standing stripe structure attained in the limit of very high velocities. Stripes in this mode undulate in a rather complex way in the experiments, showing propagative relaxation in both longitudinal and transversal modulations $[43,46]$ in a way that is qualitatively consistent with the analysis of modulational instabilities of Section 5.1. In Fig. 29 we show an example of this regime of waving stripes.

\subsection{Traveling-wave forcing in other systems and related work}

In the previous sections we have seen that the photosensitive CDIMA reaction is a suitable system to study spatiotemporal forcing in general due to the versatility of the forcing mechanism using simple optical techniques. Nevertheless, the effects here studied are potentially observable in virtually any pattern forming system undergoing a supercritical bifurcation to a spatially periodic pattern. Traditional Rayleigh-Bénard convection is one of the obvious systems that come to mind, although the procedure to introduce a traveling modulation of the control parameter is not so simple. Purely spatial forcing has been implemented again in this system very recently with an aim also at spatio-temporal forcing in Ref. [38]. 


\section{ARTICLE IN PRESS}
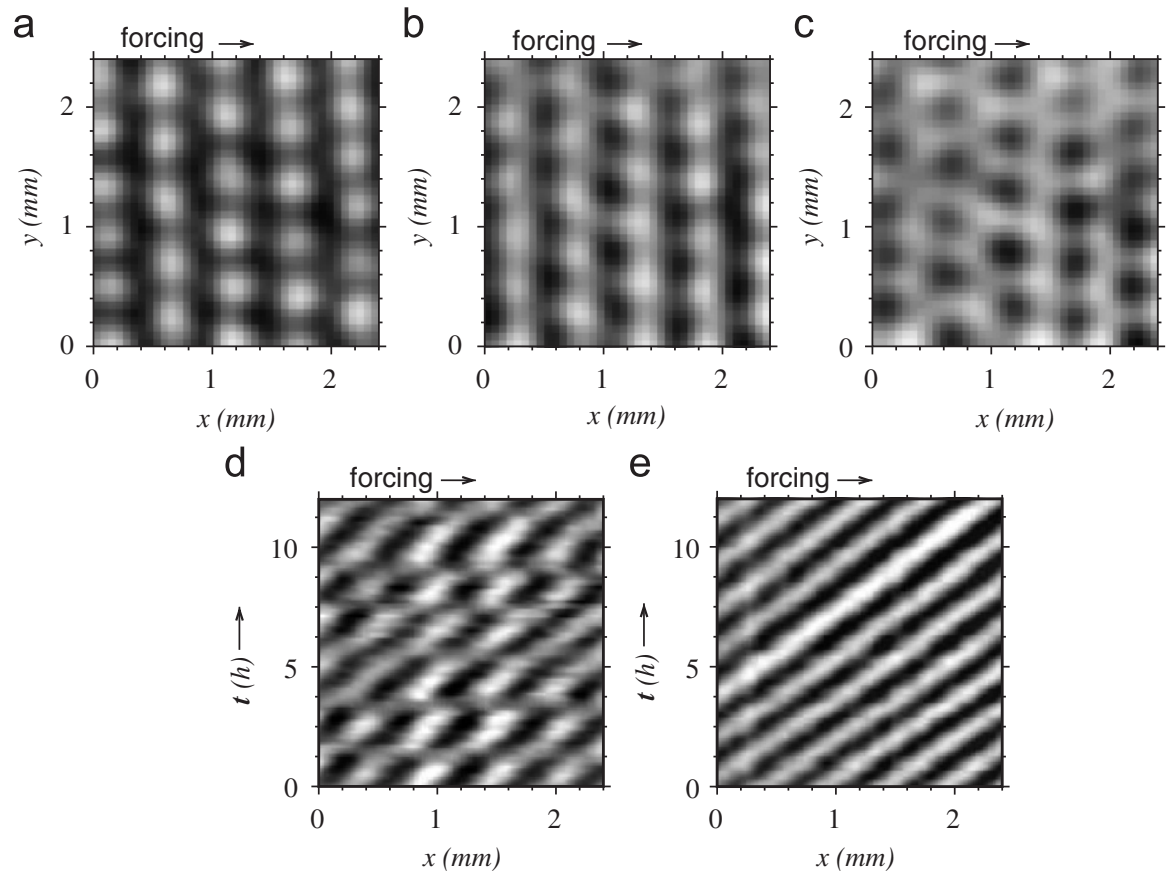

Fig. 27. Oscillating hexagons for a forcing velocity of $v_{f}=0.28 \mathrm{~mm} / \mathrm{h}$. The pattern oscillates between lattices of white spots (in (a)), black spots (c), and an intermediate state (in (b)) where both lattices are interlaced. A space-time plot is shown in (d), whereas (e) visualizes the corresponding experimental forcing pattern. (Adapted from [42].)
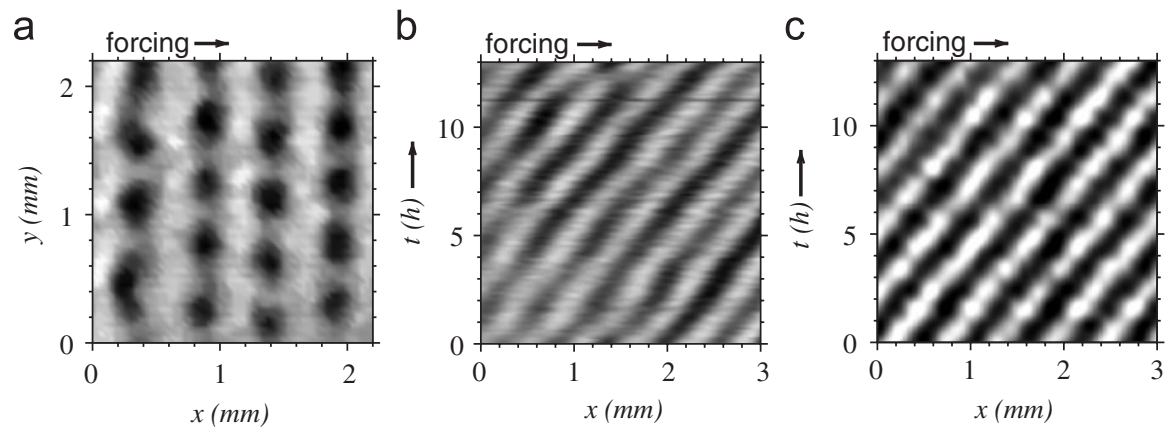

Fig. 28. Entrained hexagons appearing for a forcing velocity of $v_{f}=0.16 \mathrm{~mm} / \mathrm{h}$. In (a) we show a snapshot and in (b) the corresponding spatio-temporal plot of a cut along the horizontal direction. In (c) we show the forcing applied to the experiment. (Adapted from [42].)

Other traditional pattern-forming systems [1] may be more amenable to arbitrary spatio-temporal modulations of control parameters, for instance in the areas of electroconvection of nematic liquid crystals, nonlinear optics, reaction-diffusion systems, or phase separation. Those systems will in general cover different parameter regimes of the corresponding amplitude equations and therefore may enrich the different dynamical behaviors with yet unsuspected phenomena. In some cases, these other systems may introduce new physical ingredients which dramatically change the present scenario, for instance if the presence of linear and nonlinear dispersive effects lead to the complex Ginzburg-Landau equation as the core amplitude equation where forcing is introduced, or if the system has no intrinsic wavelength, such as in phase separation.

From a theoretical point of view, it may be useful to study some of the usual simple model equations for pattern formation, such as the Swift-Hohenberg model and its variants [1], the Proctor-Sivashinsky model, or the CahnHilliard equation for phase separation. The two latter examples have already been studied in detail for spatial forcing, 
a

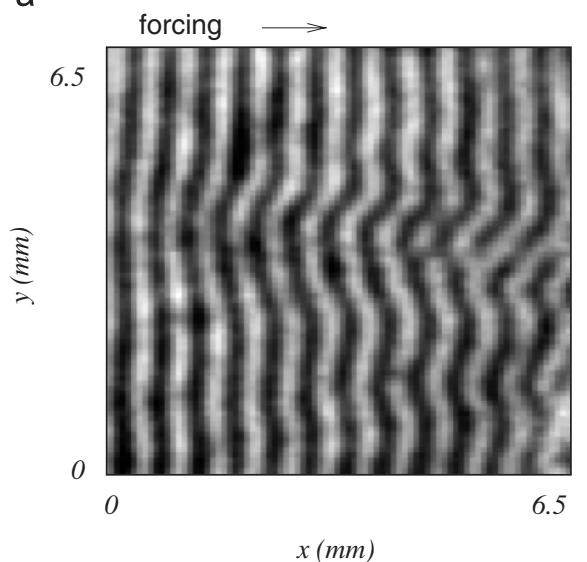

b

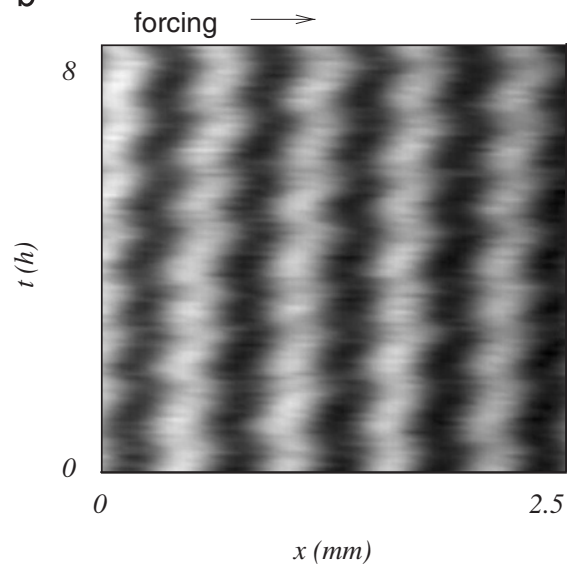

Fig. 29. Waving stripes appearing for a forcing velocity of $v_{f}=0.55 \mathrm{~mm} / \mathrm{h}$. In (a) the area shown corresponds to $6.5 \times 6.5 \mathrm{~mm}$ and the wavelength is $0.46 \mathrm{~mm}$. (b) is the corresponding spatio-temporal plot. (Adapted from [43].)

respectively, in Ref. [31] and more recently in Ref. [74]. The study of different prototype model equations such as the above has the advantage that the effects of the different physical ingredients may be more easily isolated, and at the same time, the parameters of the corresponding amplitude equations are more readily accessible. One particular feature to be explored, for instance, is the case of patterns with other symmetries (squares or rectangles) [50]. This would require deriving new sets of amplitude equations for the corresponding symmetries. Finally, the description of pattern dynamics in terms of the above model equations allows for the study of the spatial competition of different types of solutions which may coexist, for instance as observed in some experiments on the CDIMA reaction, where domains with different types of hexagons are seen to compete [45].

In this general context of spatio-temporal forcing of pattern forming systems we will now briefly review contributions from other authors which are relevant to the present discussion. As mentioned above, the introduction of spatio-temporal forcing in oscillatory systems is a clear step further to continue the present analysis. This has already been pioneered by other authors. As a matter of fact, to our knowledge, the first study of spatio-temporal forcing is that of Utzny et al. [41], which was done precisely in the more complicated case of a Hopf unstable media. Previous studies of stochastic external forcing with light in oscillatory or excitable systems had preceded that work, but the focus there was rather different, on noise effects $[64,65]$. Utzny et al. chose a forcing which was periodic in time, but which additionally depended on one space coordinate. The corresponding amplitude equation was the complex Ginzburg-Landau equation (with linear and nonlinear dispersion), and where the effect of the spatially dependent forcing was reflected by a spatially dependent coefficient of the resonance term. There they described the case of 2:1 temporal resonance between the internal and external frequencies and a periodic space dependence of the forcing. The pattern then appeared to be spatially harmonic (with the periodicity of the forcing) or subharmonic (twice the wavelength of the forcing). Both patterns could coexist for a certain range of parameters and propagating defects between regions of both types were observed.

A more recent extension of the work described in this review into oscillatory systems is the study of the travelingwave forcing of traveling waves such as can be found in Hopf unstable media where the basic instability is to a mode with non-zero wave-number and a non-zero frequency. This work constitutes a direct link between the cases of temporal and spatial resonance and was initiated by Ohta and co-workers [52-54] in the context of phase separation coupled to chemical reactions. In general terms, one can then expect both effects from the complex nature of all coefficients in amplitude [15-17] as well as the effects caused by a spatial mismatch. The case of traveling-stripe forcing of a system which supports traveling stripe solutions is a very promising problem.

Another extension of our problem has been reported by Schuler et al. [50]. This work is much closer to the present study and deals with non-oscillatory systems, but addresses patterns with different symmetries. A traveling-stripe forcing at 2:1 resonance is considered in an anisotropic pattern-forming system, such as for thermal or electroconvection of nematic liquid crystals. In this case, stripes (rolls) compete with rectangular patterns. 
An interesting application of spatio-temporal forcing has also been introduced recently by Zykov et al. [51]. They apply traveling-wave forcing to an excitable medium exhibiting rotating spirals. The aim of this study is to demonstrate that the motion of the tip of the spiral can be controlled by the forcing. For a resonance between the velocity of the forcing wave and a circular spiral tip motion, a linear motion of the spiral tip is obtained. Outside the resonant tongue a complex cycloidal-like motion occurs.

Finally, let us mention a different type of spatio-temporal forcing which has been studied and has already displayed rather puzzling responses of the systems. This consists of experiments where some boundary of the system is moving with a finite velocity (for instance a mask producing a local inhomogeneous illumination in the CDIMA reaction, which is traveling) [55-57]. The resulting reaction-diffusion-advection system has shown also a rich variety of surprising and yet unexplained phenomena, such as those reported for instance in Ref. [56].

\section{Conclusions. Open questions and future directions}

In this paper we have reviewed and pursued further some recent work on a simple type of spatio-temporal forcing in the form of a traveling-wave modulation of a control parameter. Most of this research was devoted to systems close to an instability to a stationary and spatially periodic pattern. While the spatial periodicity of the forcing generates the basic resonance, the time dependence and the deviation from exact spatial resonance produce a wealth of different scenarios and transitions.

We have presented a unified framework of these phenomena in terms of amplitude equations for both $1 \mathrm{~d}$ and $2 \mathrm{~d}$ patterns. The approach based on amplitude equations emphasizes the universal character of many of the phenomena here unveiled. We have shown that, regardless of spatial dimension and of the type of patterns that may be generated, for each order of resonance, there is a common (zero-dimensional) dynamical system underlying the phenomenon of traveling-stripe resonance. This underlying system has the same form as the amplitude equation describing a Hopf bifurcation under time-periodic forcing. The frequency $\omega$ introduced by the motion of the traveling-wave forcing is directly analogous to the frequency mismatch (detuning) between the Hopf bifurcation and the time-periodic forcing.

The problem of homogeneous states in $1 \mathrm{~d}$ can thus be mapped into the problem of temporal forcing. In the case of higher spatial dimension, however, the interaction between the mode resonant with the forcing and other modes through the nonlinear coupling is altered, and therefore the emergence of patterns with other symmetries (hexagons, squares, etc.) is affected and, most importantly is endowed with complex spatio-temporal dynamics. On the other hand, the presence of a wave-number mismatch $q$ in the spatial resonance breaks the remaining spatial reflection symmetry for nonhomogeneous solutions and, in combination with $\omega \neq 0$, yields inherently new phenomena that we have only partially explored.

For homogeneous solutions, we have shown that, while the primary instability to entrained stripe patterns is altered by the spatial resonance differently for different spatial resonance ratios $n: 1$, the time dependence of the forcing introduces a further transition to oscillatory homogeneous patterns. More complex behavior occurs if one allows for hexagonal patterns, which has been studied in the case $n=1$. Counterintuitively, moving stripe forcing can stabilize hexagons in parameter regimes, where unforced three-mode interaction favors stripes, as found in experiments of the CDIMA reaction.

Non-homogeneous solutions have been studied in $1 \mathrm{~d}$, where the emphasis was put on the dynamics and interactions of localized solutions (defects) such as pulses or kinks. The time dependence of the forcing has then important consequences for the motion of defects. These defects occur in spatially forced systems because of a stabilization mechanism owing to the spatial resonance term. The motion of the forcing then leads to a non-gradient dynamics of the system, typically resulting in domain walls which move with a non-trivial velocity. We have furthermore studied the effect of spatial mismatch on defects. While the mismatch introduces a competition between the intrinsic and the extrinsic periodicity, defects accommodate both components and therefore are more prominent as the mismatch is increased. The complexity of this competition is reflected by a transition to a defect interaction which oscillates with the distance of defects and which ultimately leads to the existence of stable chaotic wave trains of domain walls.

The success of this amplitude equation reduction approach was demonstrated primarily in experiments of the CDIMA reaction and in numerical simulations of a reaction-diffusion model of this system. We have described experiments supporting the existence of several transition predicted by the amplitude equation in real systems. So far, the experimental group in Santiago de Compostela has found for $n=1$ the transition to oscillatory traveling stripes, transition between hexagons and stripes depending on the forcing velocity including entrained and oscillating hexagons, and traveling defects, to name a few examples. 
The results here reported have focused in the simplest situations, as proposed in Ref. [40] and its follow-ups by the same and other groups. These first steps in the simplest possible case of spatio-temporal forcing have already unveiled a new world of nonlinear response phenomena which is yet to be explored and extended in many promising directions. On the theoretical side, interesting extensions could explore other parameter regimes of the amplitude equations; modulated striped patterns; complex dynamics of hexagonal patterns (chaotic regimes of the homogeneous amplitude equation); transition to squares or rectangular patterns in other systems; non-homogeneous solutions showing spatial competition between patterns of different symmetries; extension to other types of spatio-temporal forcing (moving boundaries, forcing with target or spiral waves, etc); and most remarkably, extension of the problem to systems supporting traveling waves, either oscillatory (with complex parameters of the amplitude equations) or excitable. On the theoretical side, one should also mention that interesting phenomena observed in experiments with the CDIMA reaction remain unexplained. On the experimental side, many predictions of our work could be directly studied in the context of the CDIMA reaction, for instance in the case of 2:1 resonance. More detailed parameter studies of defect instabilities and motion requires a good control of experimental conditions. Perhaps also the recent design of a forced Rayleigh-Bénard cell [38] may contribute further experimental validation of the current theory, and bring out new phenomena. In general, it would be interesting to explore other physical systems in which traveling-wave forcing could be easily implemented, which would relate to other regions of the amplitude-equation phase diagram, or introduce other types of patterns, for instance in the contexts of electroconvection of nematics, nonlinear optics, reaction-diffusion systems, etc.

One interesting aspect of the present study is its possible relevance in the problem of pattern control. Resonant perturbations are particularly suitable for control of dynamical patterns since a small forcing may achieve strong effects, i.e. such mechanism can work very efficiently. We have seen that resonant spatio-temporal forcing provides a natural tool for defect manipulation or for displacement of non-trivial locked states. These aspects open new possibilities in the context of the recently proposed mechanisms of information storage and transmission in nonequilibrium media [75], in which several practical applications of information processing devices have been suggested, for instance in semiconductor lasers [76] or in reaction-diffusion systems [77]. Therefore, further study of spatio-temporal forcing is not only relevant from a fundamental perspective, but may progress the efficiency and accuracy of control in complex systems and possibly result in practical applications.

\section{Acknowledgments}

This work was carried out within the framework of the E.U. Research Training Network PHYNECS (HPRN-CT2002-00312). S.R. acknowledges support from Deutsche Forschungsgemeinschaft (grant FA 350/6-1) and J.C. from the Spanish Ministry (FIS2006-03525). The work reviewed here is the result of collaboration and discussion through the years with many people. On the theoretical side we are particularly grateful to Francesc Sagués, for his driving force and significant contributions at the early stages of this research. Illuminating discussions with Werner Pesch are also acknowledged. This study would not have been the same without the crucial role of the experiments on the CDIMA reaction, which were carried out by David G. Míguez and Alberto P. Muñuzuri. Finally, the authors would like to express their deepest thanks and recognition to Lorenz Kramer, who passed away in the late stages of this work. We are all in debt with L.K. because of his eminent scientific contribution but also his human quality and personal guidance.

\section{References}

[1] M.C. Cross, P.C. Hohenberg, Rev. Mod. Phys. 65 (1993) 851.

[2] D. Walgraef, Spatio-Temporal Pattern Formation, Springer, New York, 1997.

[3] A. Pikovsky, M. Rosenblum, J. Kurths, Synchronization: A Universal Concept in Nonlinear Sciences, Cambridge University Press, 2003.

[4] A.S. Mikhailov, K. Showalter, Phys. Rep. 425 (2006) 79.

[5] P. Coullet, J. Lega, B. Houchmanzadeh, J. Lajzerowics, Phys. Rev. Lett. 65 (1990) 1352-1355.

[6] P. Coullet, K. Emilsson, Physica D 61 (1992) 119-131.

[7] V. Petrov, Q. Ouyang, H.L. Swinney, Nature 388 (1997) 655-657.

[8] C. Elphick, A. Hagberg, E. Meron, Phys. Rev. Lett. 80 (1998) 5007.

[9] A.L. Lin, M. Bertram, K. Martinez, H.L. Swinney, A. Ardalea, G.F. Carey, Phys. Rev. Lett. 84 (2000) 4240.

[10] A.L. Lin, A. Hagberg, A. Ardelea, M. Bertram, H.L. Swinney, E. Meron, Phys. Rev. E 62 (2000) 3790.

[11] H.-K. Park, Phys. Rev. Lett. 86 (2001) 1130.

[12] A. Yochelis, A. Hagberg, E. Meron, A.L. Lin, H.L. Swinney, Siam J. Appl. Dyn. Sys. 1 (2002) 236. 
[13] A. Yochelis, C. Elphick, A. Hagberg, E. Meron, Physica D 199 (2004) 201.

[14] A.L. Lin, A. Hagberg, E. Meron, H.L. Swinney, Phys. Rev. E 69 (2004) 066217.

[15] H. Chaté, A. Pikovsky, O. Rudzick, Physica 131D (1999) 17.

[16] M. Argentina, O. Rudzick, M.G. Velarde, Chaos 14 (2004) 777.

[17] O. Rudzick, A.S. Mikhailov, Phys. Rev. Lett. 96 (2006) 018302.

[18] W. Zhang, J. Viñals, J. Fluid Mech. 336 (1997) 301-330.

[19] A.K. Horvath, M. Dolnik, A.P. Muñuzuri, A.M. Zhabotinsky, I.R. Epstein, Phys. Rev. Lett. 83 (1999) $2950-2952$.

[20] M. Dolnik, A.M. Zhabotinsky, I.R. Epstein, Phys. Rev. E 63 (2001) 026101.

[21] A. Careta, F. Sagues, J. Chem. Phys. 92 (1990) 1098-1105.

[22] M. Lowe, J.P. Gollub, T.C. Lubensky, Phys. Rev. Lett. 51 (1983) 786-789.

[23] M. Lowe, J.P. Gollub, Phys. Rev. A 31 (1985) 3893-3897.

[24] M. Lowe, B.S. Albert, J.P. Gollub, J. Fluid Mech. 173 (1986) 253-272.

[25] P. Coullet, Phys. Rev. Lett. 56 (1986) 724.

[26] P. Coullet, P. Huerre, Physica D 23 (1986) 27-44.

[27] P. Coullet, D. Repaux, Europhys. Lett. 3 (1987) 573-579.

[28] P. Coullet, D. Elphick, D. Repaux, Phys. Rev. Lett. 58 (1987) 431.

[29] C. Elphick, E. Meron, E.A. Spiegel, Phys. Rev. Lett. 61 (1988) 496.

[30] P. Coullet, D. Walgraef, Europhys. Lett. 10 (1989) 525-531.

[31] L.M. Pismen, Phys. Rev. Lett. 59 (1987) 2740-2743.

[32] M. Dolnik, I. Berenstein, A.M. Zhabotinsky, I.R. Epstein, Phys. Rev. Lett. 87 (2001) 238301.

[33] L. Yang, M. Dolnik, A.M. Zhabotinsky, I.R. Epstein, Phys. Rev. Lett. 88 (2002) 208303.

[34] D. Semwogerere, M.F. Schatz, Phys. Rev. Lett. 88 (2002) 054501.

[35] R. Neubecker, A. Zimmermann, Phys. Rev. E 65 (2002) 035205.

[36] M. Henriot, J. Burguete, R. Ribotta, Phys. Rev. Lett. 91 (2003) 104501.

[37] R. Peter, M. Hilt, F. Ziebert, J. Bammert, C. Erlenkämper, N. Lorscheid, C. Weitenberg, A. Winter, M. Hammele, W. Zimmermann, Phys. Rev. E 71 (2005) 046212 .

[38] J.H. McCoy, W. Brunner, W. Pesch, E. Bodenschatz, 2006, preprint.

[39] M. Hammele, W. Zimmermann, Phys. Rev. E 73 (2006) 066211.

[40] S. Rüdiger, D.G. Míguez, A.P. Muñuzuri, F. Sagués, J. Casademunt, Phys. Rev. Lett. 90 (2003) 128301.

[41] C. Utzny, W. Zimmermann, M. Bär, Europhys. Lett. 57 (2002) 113.

[42] D.G. Míguez, E.M. Nicola, A.P. Muñuzuri, J. Casademunt, F. Sagués, L. Kramer, Phys. Rev. Lett. 93 (2004) 048303.

[43] F. Sagués, D.G. Míguez, E.M. Nicola, A.P. Munũzuri, J. Casademunt, L. Kramer, Physica D 199 (2004) 235.

[44] S. Rüdiger, J. Casademunt, L. Kramer, Disc. Cont. Dyn. Syst. B 5 (2005) 1027.

[45] D.G. Míguez, V. Pérez-Villar, A.P. Muñuzuri, Phys. Rev. E 71 (2005) 066217.

[46] I. Berenstein, A.P. Muñuzuri, Phys. Rev. E 74 (2006) 036202.

[47] S. Rüdiger, J. Casademunt, L. Kramer, Phys. Rev. Lett. (2007), to appear.

[48] P. Coullet, C. Riera, C. Tresser, Chaos 14 (2004) 193.

[49] P. Coullet, C. Toniolo, C. Tresser, Chaos 14 (2004) 839.

[50] S. Schuler, M. Hammele, W. Zimmermann, Eur. Phys. J. B 42 (2004) 591.

[51] S. Zykov, V.S. Zykov, V. Davydov, Europhys. Lett. 73 (2006) 335-341.

[52] T. Ohta, H. Tokuda, Phys. Rev. E 72 (2005) 046216.

[53] H. Tokuda, T. Ohta, Progr. Theor. Phys. 161 (Suppl.) (2006) 368.

[54] H. Tokuda, T. Ohta, J. Phys. Soc. Japan 75 (2006) 064005.

[55] V.K. Vanag, I.R. Epstein, Phys. Rev. E 67 (2003) 066219.

[56] D.G. Míguez, M. Dolnik, A.P. Muñuzuri, L. Kramer, Phys. Rev. Lett. 96 (2006) 048304.

[57] D.G. Míguez, R.A. Satnoianu, A.P. Muñuzuri, Phys. Rev. E 73 (2006) 025201(R).

[58] I. Lengyel, G. Rábai, I.R. Epstein, J. Am. Chem. Soc. 112 (1990) 4606-4607.

[59] A.P. Muñuzuri, M. Dolnik, A.M. Zhabotinsky, I.R. Epstein, J. Am. Chem. Soc. 121 (1999) 8065-8069.

[60] A.M. Turing, Philos. Trans. R. Soc. London B 237 (1952) 37.

[61] V. Castets, E. Dulos, J. Boissonade, P. De Kepper, Phys. Rev. Lett. 64 (1990) 2953-2956.

[62] Q. Ouyang, H.L. Swinney, Nature 352 (1991) 610-612.

[63] R. Kapral, K. Showalter (Eds.), Chemical Waves and Patterns, Kluwer Academic Publishers, Dordrecht, 1995.

[64] S. Kadar, J. Wang, K. Showalter, Nature 391 (1998) 770.

[65] I. Sendiña-Nadal, S. Alonso, V. Pérez-Muñuzuri, M. Gómez-Gesteira, V. Pérez-Villar, L. Ramírez-Piscina, J. Casademunt, J.M. Sancho, F. Sagués, Phys. Rev. Lett. 84 (2000) 2734

[66] J.M. Gambaudo, J. Differential Equations 65 (1985) 172-199.

[67] S.H. Strogatz, Nonlinear Dynamics and Chaos, Addison-Wesley, Reading, MA, 1994.

[68] E.J. Doedel, A.R. Champneys, T.F. Fairgrieve, Yu.A. Kuznetsov, B. Sandstede, X. Wang (1997) AUTO97 (with HomCont), Technical Report, Concordia University, 1997.

[69] B. Ermentrout, Simulating, Analyzing, and Animating Dynamical Systems: A Guide to Xppaut for Researchers and Students, SIAM, 2002.

[70] D.V. Skryabin, A. Yulin, D. Michaelis, W.J. Firth, G.-L. Oppo, U. Peschel, F. Lederer, Phys. Rev. E 64 (2001) 056618.

[71] L.M. Pismen, Chaotic wave trains, Phys. Rev. A 39 (1989) 6604. 
[72] M. Levi, F. Hoppenstedt, W. Miranker, Quart. Appl. Math. 35 (1978) 167.

[73] R. Kupferman, M. Kaiser, Z. Schuss, E. Ben-Jacob, Phys. Rev. A 45 (1992) 745.

[74] A. Krekhov, L. Kramer, Phys. Rev. E 70 (2004) 061801.

[75] P. Coullet, C. Riera, C. Tresser, Chaos 14 (2004) 193-198.

[76] S. Barland, J.R. Tredicce, M. Brambilla, L.A. Lugiato, S. Balle, M. Giudici, T. Maggipinto, L. Spinelli, G. Tissoni, T. Knodl, M. Miller, R. Jager, Nature 419 (2002) 699-702.

[77] A. Kaminaga, V.K. Vanag, I.R. Epstein, Angew. Chem. Int. Ed. 45 (2006) 3087-3089. 